Article

\title{
Application of the DC Offset Cancellation Method and S Transform to Gearbox Fault Diagnosis
}

\author{
Xinghui Zhang ${ }^{1, *}$, Jianmin Zhao ${ }^{1}$, Rusmir Bajrić ${ }^{2}$ and Liangliang Wang ${ }^{1}$ \\ 1 The management department of Mechanical Engineering College, Shijiazhuang 050003, China; \\ jm_zhao@hotmail.com (J.Z.); wanglsjz@163.com (L.W.) \\ 2 Public Enterprise Elektroprivreda BiH, Coal Mines Kreka, Tuzla 75000, Bosnia and Herzegovina; \\ rusmir.bajric@gmail.com or rusmir.bajric@kreka.ba \\ * Correspondence: dynamicbnt@163.com; Tel.: +86-186-0329-2514
}

Academic Editor: David He

Received: 31 December 2016; Accepted: 9 February 2017; Published: 20 February 2017

\begin{abstract}
In this paper, the direct current (DC) offset cancellation and S transform-based diagnosis method is verified using three case studies. For DC offset cancellation, correlated kurtosis (CK) is used instead of the cross-correlation coefficient in order to determine the optimal iteration number. Compared to the cross-correlation coefficient, CK enhances the DC offset cancellation ability enormously because of its excellent periodic impulse signal detection ability. Here, it has been proven experimentally that it can effectively diagnose the implanted bearing fault. However, the proposed method is less effective in the case of simultaneously present bearing and gear faults, especially for extremely weak bearing faults. In this circumstance, the iteration number of DC offset cancellation is determined directly by the high-speed shaft gear mesh frequency order. For the planetary gearbox, the application of the proposed method differs from the fixed-axis gearbox, because of its complex structure. For those small fault frequency parts, such as planet gear and ring gear, the DC offset cancellation's ability is less effective than for the fixed-axis gearbox. In these studies, the $S$ transform is used to display the time-frequency characteristics of the DC offset cancellation processed results; the performances are evaluated, and the discussions are given. The fault information can be more easily observed in the time-frequency contour than the frequency domain.
\end{abstract}

Keywords: planetary gearbox; fault diagnosis; predictive maintenance; deterministic component cancellation; $\mathrm{S}$ transform

\section{Introduction}

To stay competitive, many companies all over the world try to go beyond the costly industry standard of preventative maintenance by implementing predictive maintenance procedures. For the industry related to oil exploitation, nuclear power and wind power, predictive maintenance procedures are the backbone in order to improve the operation and maintenance. Additionally, those industries for operation use drive trains that usually contain a gearbox as one of the key components. Vibration analysis is the most widely-used predictive maintenance procedure in gearbox predictive maintenance. In many mechanical systems, gearboxes are widely used for power transmission. Its condition is very important for the performance of the whole mechanical system. Therefore, many companies-and in the last two decades of academia also-have paid great attention to the gearbox condition monitoring. Vibration analysis can be applied very effectively to gearbox condition monitoring and predictive maintenance. In 2006, Jardine et al. [1] reviewed the machinery fault diagnosis and prognosis in the condition-based maintenance view. The authors considered fault diagnosis as an important part of condition-based maintenance. We know that the gearbox is composed of many parts, such as gears, shafts, bearings, etc. In addition, there are many types of gearbox. The most typical are the 
fixed-axis gearbox and planetary gearbox. In 2011, bearing fault diagnosis work was reviewed by Randall and Antoni [2]. Later, Ray and Upadhyay [3] reviewed the signal processing techniques used in bearing fault diagnosis. This focuses mainly on the recent developments of the fault diagnosis signal processing techniques. Then, they discussed the advantages and disadvantages of these methods used in bearing fault diagnosis. For time-frequency analysis, Feng et al. [4] reviewed more than 20 major time-frequency analysis methods used in fault diagnosis since 1990 and examined their advantages and disadvantages. As a major time-frequency analysis method, multiwavelet's development in fault diagnosis has been reviewed by Sun et al. [5]. Finally, they discussed the existing problems and future research directions. As we know, the fault mechanism of the planetary gearbox is very different from fixed-axis gearbox. Therefore, many authors pay great attention to the fault diagnosis of planetary gearboxes. Lei et al. [6] reviewed the papers emerging in the planetary gearbox fault diagnosis domain and compared the fixed-axis and planetary gearbox fault diagnosis. Finally, they pointed to the potential research topics. Through these detailed review papers, readers will have a good knowledge of gearbox fault diagnosis.

Whether for the gear or the bearing, fault will produce impulse signals with a specific period. Therefore, the main object for fault diagnosis is to detect these impulse signals. Therefore, many methods were developed to detect impulse signals. Spectral kurtosis was one such effective method used for impulse signal detection. From its proposal, it becomes an important and effective way to detect the impulse signal. Recently, Wang et al. [7] reviewed the development of spectral kurtosis and pointed out its future use in fault prognosis. Nevertheless, these periodic impulse signals (PIS) produced by fault are always overwhelmed by deterministic signals produced by the regular gear mesh and structural vibration. These deterministic signals are also called discrete frequency signals or the deterministic component. In order to detect the impulse signal more easily, it is necessary to suppress the deterministic signals. There are several methods developed to subtract the deterministic component from the original signal. The most dominant were: the autoregressive (AR) model method [8-15], time synchronous averaging [16-18], adaptive noise cancellation $[19,20]$ and the cepstrum pre-whitening method [21-23]. These four methods all have been used in the separation of the deterministic signal and PIS signal successfully. Except for these methods, a new deterministic component cancellation method was proposed, recently [24]. It is based on an iterative calculation of the signal envelope when the DC offset is cancelled before envelope calculation. The only parameter that needs to be pre-determined is the iteration number for DC offset cancellation. In the paper, a threshold of cross-correlation coefficient interval of $[0.9,0.95]$ was selected to judge the proper number of envelope calculations. The selected correlation coefficient interval was efficient for the examples in the paper, but this threshold method is not a proper way to acquire the most powerful PIS. Sometimes, a greater iteration number will distort the waveform of PIS and lead to the misdiagnosis of faults. In order to resolve this problem, correlated kurtosis $(\mathrm{CK})$ is proposed to be used in determining when to stop the iteration for envelope calculation [25]. This indicator can detect the PIS properly. However, CK is efficient only with strong enough PIS. When the PIS is very weak, CK is inefficient due to the inability to correctly detect the PIS. Therefore, in the case of very weak PIS, it is very difficult to determine the appropriate iteration number for envelope calculation.

Time frequency analysis methods, such as short time Fourier transform, the Wigner-Ville distribution and wavelet transform, were widely used in rotating machinery fault diagnosis. However, they all have some demerits. Compared to these time frequency analysis methods, the S transform can greatly avoid the existing deficiencies of the above three methods [26]. Therefore, it can be used to display the time-frequency characteristics of the separation results of the deterministic component and the random component. In addition, fault features can be extracted from the resulting signal of the $\mathrm{S}$ transform to track the degradation of the component of interest. From the time-frequency contour produced by the $\mathrm{S}$ transform, one can distinguish the different faults more easily; or it can be used as the input for the deep learning methods to intelligent fault classification. 
The above discussions show the need to use CK to optimize the iteration number of the envelope calculation with strong enough PIS. This can suppress the deterministic component effectively and properly. The $\mathrm{S}$ transform advantages inspire us to diagnose faults of the gearbox component by separation of the results of the deterministic component and the random component. The method is validated using the experimental datasets collected from a lab AC motor faulty bearing; a lab fixed-axis gearbox faulty bearing and gear and a lab planetary gearbox faulty sun, planet and ring gear. Experimental case studies will benefit the application of this novel method and will also provide a detailed guide for engineers in the industrial application. In addition, validation has been conducted using both intentionally-implanted bearing and gear damage data and naturally-developed distributed gear and bearing wear data under constant motor speed and various loads.

Based on the above-mentioned literature and analysis, the main contributions of this paper can be summarized as follows: (1) instead of the cross-correlation coefficient, correlated kurtosis was used as a criterion to estimate the optimal iteration number of DC offset cancellation, since CK has the superior ability to detect the PIS signal and is more robust to the noise interference; (2) when PIS is very weak compared with the deterministic signal or existing bearing fault and gear fault simultaneously, even CK cannot detect weak PIS produced by the bearing fault effectively; in this circumstance, the maximum order of high-speed shaft mesh frequency was used as the iteration number of DC offset cancellation; (3) because of several existing merits, the $S$ transform was used to combine with DC offset cancellation to display the time-frequency characteristic of various gearbox faults; it is very efficient and intuitive to find faults through time-frequency contours; in addition, it could be used for deep learning-based fault classification; (4) through the planetary gearbox case study, it was found that DC offset cancellation is less effective for those small fault frequency signals, such as the planet gear, ring gear, carrier, etc.

Compared to the DC offset cancellation used in [24], the advantage of this study is that it used CK instead of the cross-correlation coefficient to determine the optimal iteration number of DC offset cancellation. According to the CK's value, it is more corrective to select the iteration number because it can detect PIS more accurately than the cross-correlation coefficient and is not easily interfered with by impulse-like noise. However, the disadvantage of this method is that it is less effective when the PIS is very weak. For the $S$ transform, the advantages are that it can display the fault information more fruitfully and intuitively than the frequency domain. However, for some small fault frequencies, it is difficult to display them in the time-frequency contour because this needs a greater sampling length and will consume more computational resources, especially requiring a high ability graphic processing unit.

The remainder part of the paper is organized as follows. In Section 2, a novel pre-whitening method and the S transform are briefly introduced. In Section 3, the experimental case study of an implanted bearing outer race fault is presented. In Section 4, the experimental case study of bearing and gear fault diagnosis in the gearbox is presented and explained in detail. In Section 5, the experimental case study of the planetary gearbox fault diagnosis using the proposed method is presented. In Section 6, the effectiveness and existing problem of the novel pre-whitening method are discussed. Finally, Section 7 concludes the work.

\section{Novel Pre-Whitening Method and S Transform}

\subsection{Novel Pre-Whitening Method}

The vibration signal acquired from a gearbox is usually complex, containing many different signal components. Generally speaking, deterministic components (also called discrete frequency) represent signals produced by gear meshing, shaft bending, shaft misalignment, etc. However, random components, also called PIS, usually represent signals produced by gear or bearing faults. Traditionally, many scholars represent random components as signals of bearing faults. In this sense, when a gear has faults, such as a chipped tooth, a cracked tooth and wear of the tooth face, the meshing 
will change. Every time the faulty tooth meshes, it produces impulse signals. If the gear has severe faults, the transmission ratio will vary also. Impulse signals induced by the faulty gear are very different with the signals induced by the normal gear. The PIS produced by the faulty gear also has a random property, such as the faulty bearing, because the gear faults usually produce impulse vibration signals. Therefore, signals produced by the gear fault also belong to the random components. It is now well established that the impact produced by the local gear fault is non-stationary in nature, and conventional signal processing approaches are inappropriate; and local tooth damage (i.e., fatigue crack, pitting, etc.) produces sharp transients in the vibration signature, which can be classified as non-stationary, non-linear and non-Gaussian in nature [20]. In this content, signals collected from the gearbox can be represented as:

$$
x(t)=u(t)+f(t)
$$

where $u(t)$ denotes the deterministic components and $f(t)$ denotes random components induced by the gear or bearing fault. This is under the assumption that the rotating speed is constant or can be transformed to be constant in the angular domain by the assistance of the tachometer signal. The envelope signal can be represented as:

$$
y(t)=x(t)+j \hat{x}(t)=u(t)+f(t)+j[\hat{u}(t)+\hat{f}(t)]
$$

Because the squared envelope has a bigger SNR than the envelope alone, the squared envelope of the hybrid signal can be calculated as:

$$
\begin{gathered}
|y(t)|^{2}=x(t)^{2}+\hat{x}(t)^{2} \\
|y(t)|^{2}=u(t)^{2}+f(t)^{2}+2 u(t) f(t)+\hat{u}(t)^{2}+\hat{f}(t)^{2}+2 \hat{u}(t) \hat{f}(t)
\end{gathered}
$$

Then, Ming et al. [24] derived the analytic forms of the squared envelope and the squared envelope spectrum. They found that the primary energy of the multi-component signal was shifted to the direct current (DC) offset of the envelope signal. Both the deterministic component and the random component are reserved in the envelope of the multi-component signal when the DC offset has not been cancelled. If the DC offset is cancelled, the cross-terms of different mono-components would dominate the envelope. However, the cross-terms of the deterministic component have one fewer harmonic than the original ones. In addition, all harmonics of cross-terms between deterministic and random component shift to low band direction with different distances. That is to say, one DC offset elimination operation can subtract one harmonic of the cross-terms of the deterministic component from the envelope signal. If we repeat this process, the deterministic component would be suppressed efficiently. According to this theory, they proposed a novel deterministic component cancellation method. The detail procedure is illustrated in Figure 1.

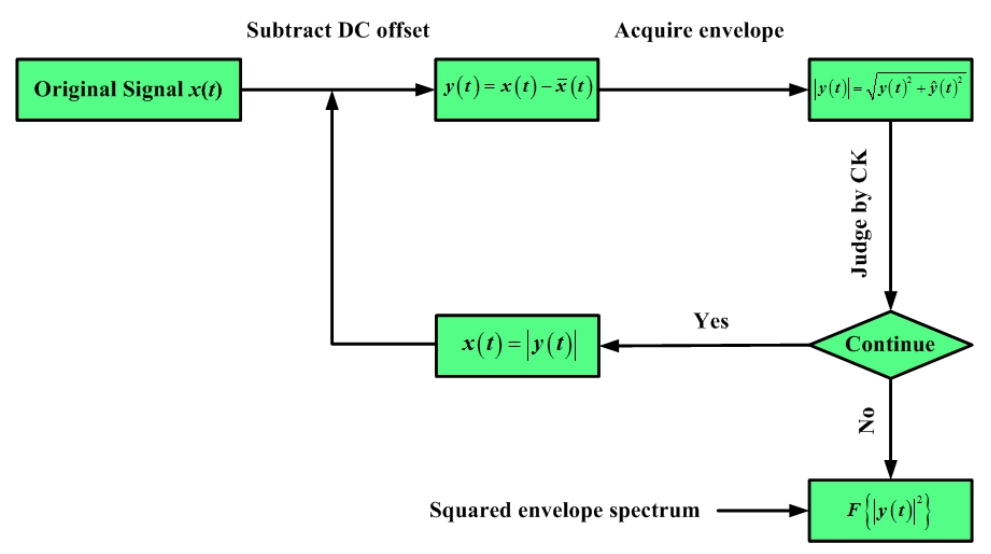

Figure 1. Framework for the deterministic component cancellation method. 
According to [24], the detailed process can be depicted as follows:

(1) Cancel the DC offset from the original multi-component signal $x(t)$, and calculate its envelope denoted as $\mathrm{H}^{k}[x(t)] ; k=1,2, \ldots, \mathrm{H}^{k}[x(t)]$ represent the $k$-th envelope obtained by Hilbert transform. Specifically, $\mathrm{H}^{\circ}[x(t)]=x(t)$.

(2) Calculate the correlated kurtosis of the envelope signal using the following equation:

$$
\text { Correlated kurtosis of } M-\text { shift }=C K_{M}(\tau)=\frac{\sum_{t=1}^{N}\left(\prod_{m=0}^{M} y(t-m \tau)\right)^{2}}{\left(\sum_{t=1}^{N} y(t)^{2}\right)^{M+1}}
$$

$y(t)$ is the input signal. $\tau$ is the interesting period of the fault. $N$ is the number of samples of input signal $y(t)$. If $\tau=0$ and $M=1$, then it gives the traditional kurtosis and can be used to detect the specific PIS. For example, if the desired fault frequency is $50 \mathrm{~Hz}$ and the sampling frequency is $10,000 \mathrm{~Hz}$, the value of $\tau$ will be 200 samples.

(3) Determine the optimal iteration number according to the $\mathrm{CK}$ value. A high $\mathrm{CK}$ value denotes the strong PIS.

(4) When the optimal iteration number is acquired, then the squared envelope spectrum of the resulting envelope signal can be acquired as follows:

$$
\begin{gathered}
|y(t)|^{2}=<\mathrm{H}^{k-1}[x(t)], \mathrm{H}^{k}[x(t)]> \\
F\left(|y(t)|^{2}\right)=F\left\{<\mathrm{H}^{k-1}[x(t)], \mathrm{H}^{k}[x(t)]>\right\}
\end{gathered}
$$

where $k$ denotes the optimal iteration number of the proposed method.

In the original paper, the iteration number is judged by following two steps as a substitute of Steps (2) and (3). First, calculate the cross-correlation coefficient of the adjacent envelopes/signals using the following equation:

$$
\mu_{k}=\frac{\left|2<\mathrm{H}^{k-1}[x(t)], \mathrm{H}^{k}[x(t)]>\right|}{<\mathrm{H}^{k-1}[x(t)], \mathrm{H}^{k-1}[x(t)]>+<\mathrm{H}^{k}[x(t)], \mathrm{H}^{k}[x(t)]>}
$$

where $<,>$ denotes the inner product operation. According to the Cauchy-Schwarz inequality, cross-correlation coefficient $\mu_{k}, k=1,2,3, \ldots$, is in interval [0,1]. When these two signals are everywhere equal, this indicator may be 1.0.

Second, a hard threshold $\lambda$ is set. If $\mu_{k} \geq \lambda$, the iteration is terminated. Through this process, the deterministic component can be adaptively subtracted by cancellation of the DC offset from the envelope.

\section{2. $S$ Transform}

The S transform combines the separate strengths of the STFT and wavelet transforms [27] and has provided an alternative approach to process the non-stationary signals generated by mechanical systems [28].

Suppose the short time Fourier transform (STFT) of signal $y(t)$ is as below:

$$
\operatorname{STFT}(\tau, f)=\int_{-\infty}^{\infty} y(t) g(\tau-t) e^{-j 2 \pi f t} d t
$$

where $\tau$ and $f$ denote the time of spectral localization and Fourier frequency, respectively, and $g(t)$ denotes a window function. 
The $\mathrm{S}$ transform can be derived from (9) by replacing the window function $g(t)$ with the Gaussian function, depicted as:

$$
g(t)=\frac{|f|}{\sqrt{2 \pi}} e^{-\frac{t^{2} f^{2}}{2}}
$$

Then, the $\mathrm{S}$ transform is defined as:

$$
S(\tau, f)=\int_{-\infty}^{\infty} y(t) \frac{|f|}{\sqrt{2 \pi}} e^{-\frac{(\tau-t)^{2} f^{2}}{2}} e^{-j 2 \pi f t} d t
$$

Actually, the $\mathrm{S}$ transform is a special case of STFT with the Gaussian window function.

If the continuous wavelet transform (CWT) is:

$$
W(\tau, d)=\int_{-\infty}^{\infty} y(t) \omega(t-\tau, d) d t
$$

where $d$ denotes the "width" of wavelet $w(t, d)$, and thus, it controls the resolution; and $w(t, d)$ denotes a scaled copy of the fundamental mother wavelet.

Then, the $S$ transform is a CWT with a specific mother wavelet multiplied by the phase factor:

$$
S(\tau, f)=e^{-j 2 \pi f t} W(\tau, d)
$$

where the mother wavelet is:

$$
\omega(t, f)=\frac{|f|}{\sqrt{2 \pi}} e^{-\frac{t^{2} f^{2}}{2}} e^{-j 2 \pi f t}
$$

Note that the factor $d$ is the inverse of the frequency $f$.

Compared with STFT and CWT, the S transform has the following merits:

(1) The window length used in STFT is a fixed value. On the contrary, the window length used in the $S$ transform is a function of time and frequency. In other words, the window length is adaptive. In the time domain, the window will be wider for low frequencies and will be narrower for high frequencies. This will provide better localization in the frequency domain for low frequencies and provide better localization in the time domain for higher frequencies. These characteristics are very similar to the wavelet transform.

(2) The great difference of the $S$ transform and the wavelet transform is the different presentation of the time-frequency characteristic. The $S$ transform represents signal features in the time-frequency contour. However, the wavelet transform represents signal features in the time-scale contour. Because the $S$ transform uses frequency as a variable, this allows it to have a direct connection and avoids the error in the frequency estimation.

The most important merit of the $\mathrm{S}$ transform is the preservation of both the amplitude and phase information. However, wavelet coefficients only contain amplitude information. Because of these merits, the $S$ transform is used for mechanical fault diagnosis combined with the novel deterministic component cancellation method.

\section{Novel Proposed Method Application for Implanted Bearing Fault Diagnosis}

The vibration fault dataset used in this case study was obtained from the Mechanical Failures Prevention Group (MFPT), assembled and prepared on behalf of MFPT by Eric Bechhoefer [29]. Only the outer race faults are considered for evaluation and discussion in this research. The test bearings, which support the motor shaft, are radial ball bearings produced by RBC NICE with the following parameters: roller diameter 0.235 inch, pitch diameter 1.245 inch, number of elements 8 and the contact angle of $0^{\circ}$. In this case study dataset with a $25-\mathrm{Hz}$ input shaft rate, a $48,828-\mathrm{Hz}$ sampling frequency, a 3-s sampling duration and a 25-lbs. load are used. If we define the four bearing fault frequencies 
as ball pass frequency inner race (BPFI), ball pass frequency outer race (BPFO), ball spin frequency (BSF) and fundamental train frequency (FTF), fault frequencies could be calculated according to the geometric parameters [30]. Four fault frequencies under the shaft rate of $25 \mathrm{~Hz}$ are: $\mathrm{BPFO}(81.12 \mathrm{~Hz})$, BPFI $(118.88 \mathrm{~Hz})$, BSF $(63.86 \mathrm{~Hz})$ and FTF $(10.14 \mathrm{~Hz})$.

According to the framework in Figure 1, the DC offset is gradually cancelled. First, the process is repeated 100 times, and $\mathrm{CK}$ and cross-correlated coefficient $\mu$ are used to judge the termination of the iteration indicators. These two indicators, variation vs. iteration number, are illustrated in Figure 2 . The value of $\mu$ increases, as can be seen, with the iteration number, but up to the 100th iteration. However, it cannot attain the threshold defined in [24], which is [0.9, 0.95]. For the CK value, it increases at the beginning up to a high value, and then, it decreases. After the 15th iteration, it continues to increase. Two local optimal values were noticed during the 100 iteration process. Iteration 5 is the first one and the second one Iteration 85. Figure 3 illustrates the squared envelope and its spectrum of the DC offset cancellation after the fifth iteration. The squared envelope and its spectrum of DC offset cancellation after the 100th iteration are shown in Figure 4. Figure 4 illustrates that normal envelope signals are distorted by an excess of DC offset cancellation. Moreover, the PIS of the squared envelope signal after the fifth DC offset cancellation is stronger than the original signal. Iteration 5 is the local optimum of the CK value. However, CK can detect the PIS signal when it is more intense than the other components. If the PIS are weak or not contained in the envelope signal, CK would not work, and its high value cannot denote the strong PIS. In this case, after the fifth iteration, the envelope signal is corrupted by excess DC offset cancellation operation. Actually, the CK values after the fifth iteration cannot denote the strength of PIS.
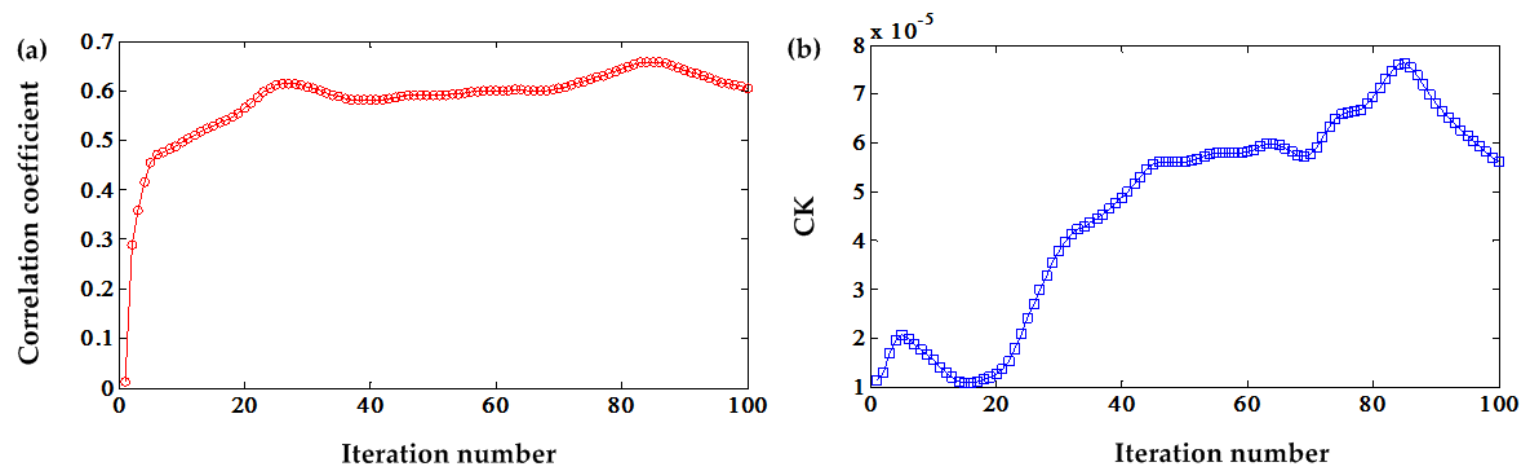

Figure 2. (a) Cross-correlation coefficient variation vs. iteration number; (b) correlated kurtosis (CK) value vs. iteration number.
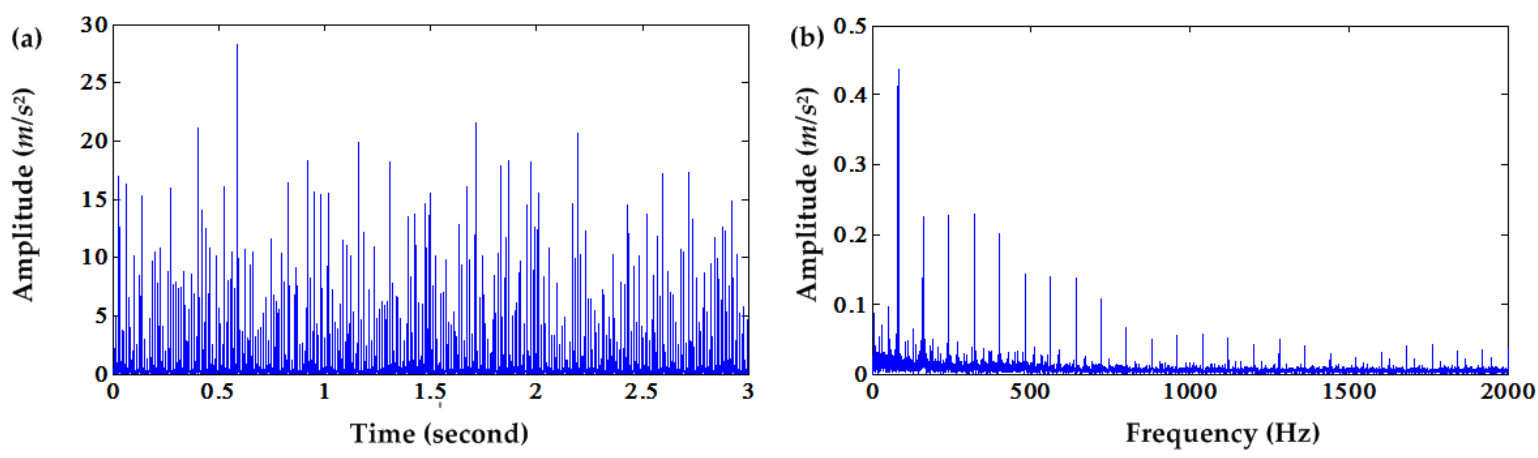

Figure 3. (a) Squared envelope signal after the fifth DC offset cancellation; (b) squared envelope spectrum after the fifth DC offset cancellation. 

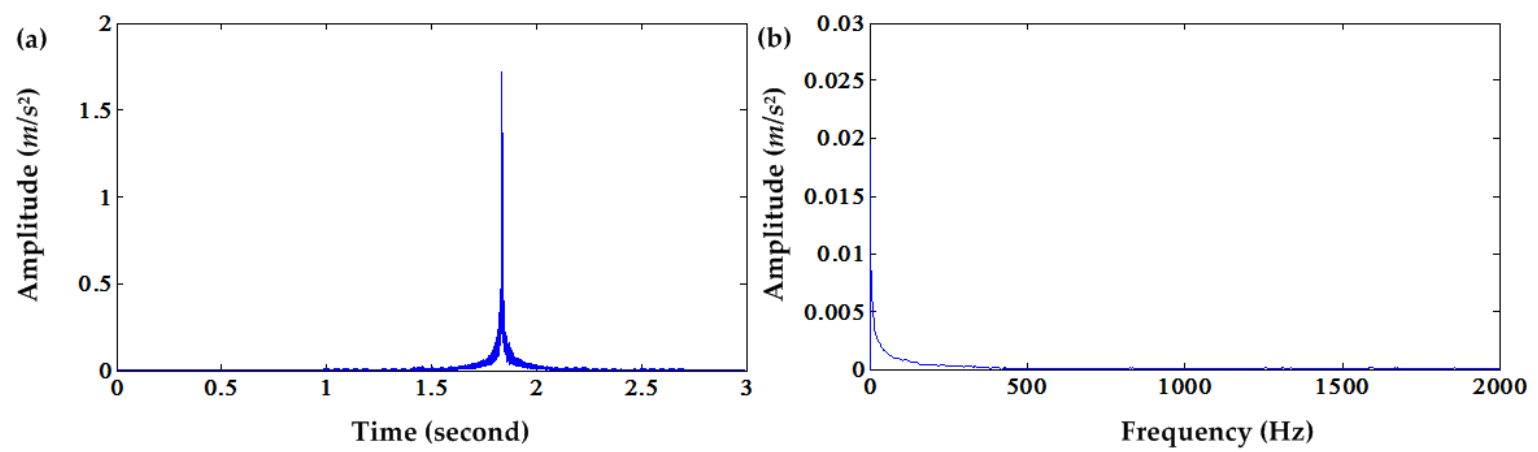

Figure 4. (a) Squared envelope signal after the 100th DC offset cancellation; (b) squared envelope spectrum after the 100th DC offset cancellation.

In order to observe the effect of DC offset cancellation from the time-frequency domain and compare it with the traditional envelope analysis, the $S$ transform is applied to the squared envelope signal after the DC offset cancellation and the envelope signal of the original signal. The results are shown in Figures 5 and 6. From the local amplification of these two time-frequency contours, the obvious BPFO can be seen in these figures. However, the time-frequency contour of the envelope of the original signal shown in Figure 5a does not have an obvious BPFO contour, whereas the time-frequency contour of the squared envelope signal after the fifth DC offset cancellation has an obvious BPFO contour. This demonstrates that the DC offset cancellation suppresses the deterministic components and enables PIS clearly enough.
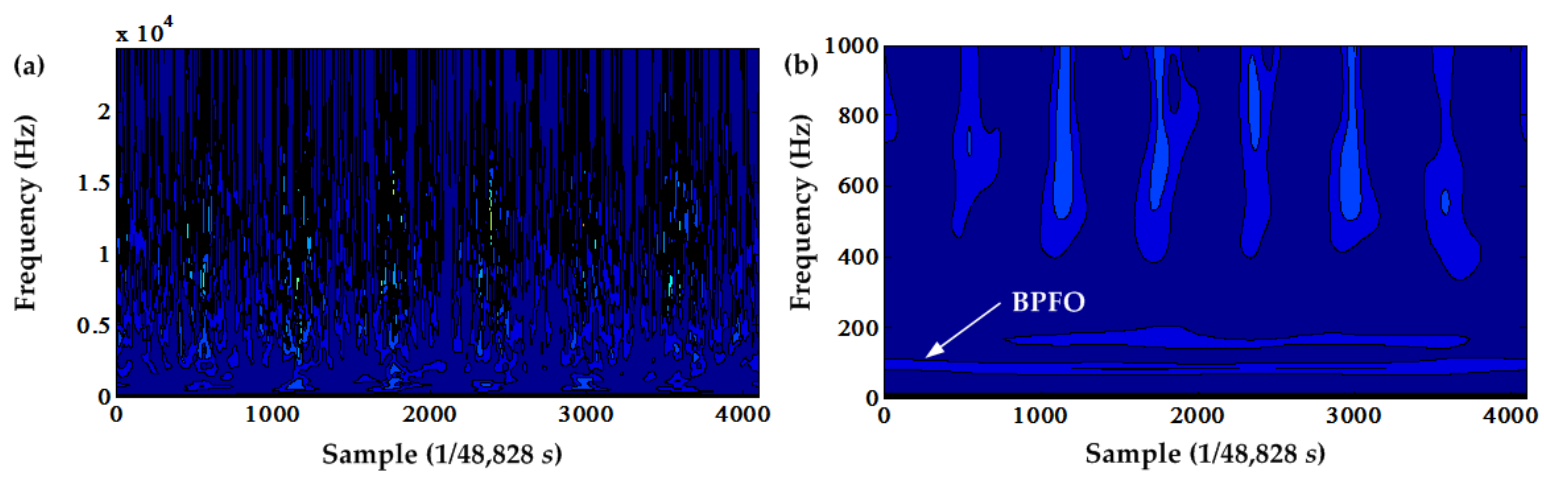

Figure 5. (a) Time-frequency contour of the envelope signal of the original bearing fault signal; (b) local amplification of (a) between 0 and $1000 \mathrm{~Hz}$. BPFO, ball pass frequency outer race.
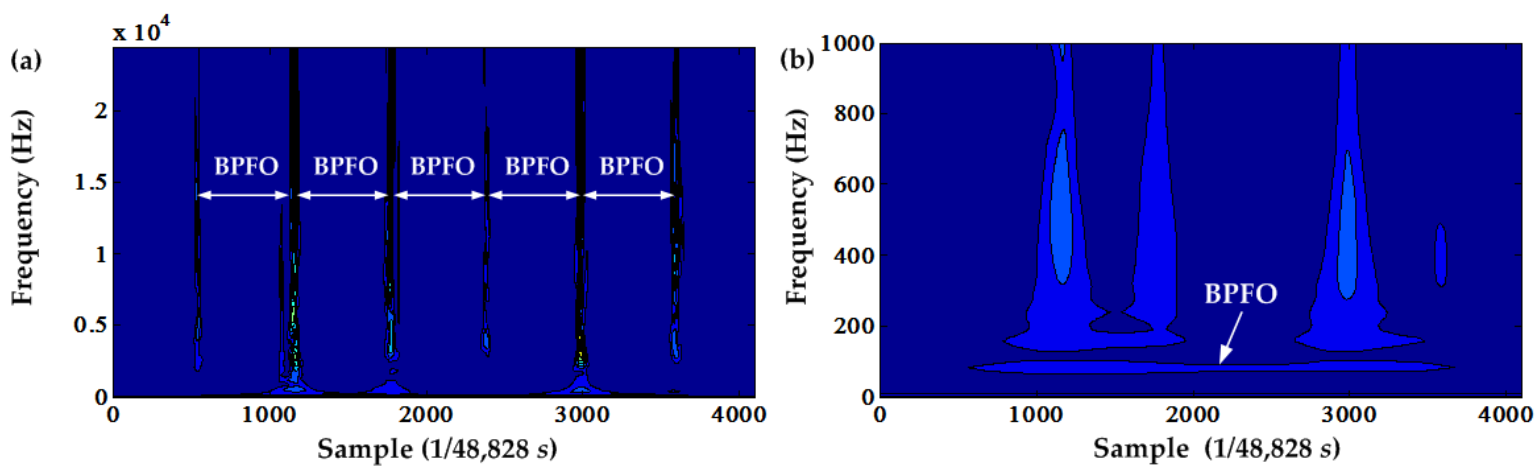

Figure 6. (a) Time-frequency contour of the square envelope signal after the fifth DC offset cancellation; (b) local amplification of (a) between 0 and $1000 \mathrm{~Hz}$. 


\section{Novel Proposed Method Application for Naturally-Developed Fault Diagnosis}

For the novel method application, experimental signals are collected from a lab fixed-axis gearbox test rig with naturally-developed bearing and gear faults. The dataset for testing high-speed (HS) shaft gear and bearings is from an end of life test case with an overall duration of $548 \mathrm{~h}$. Detail end of life test case information can be found in [31]. The intermediate speed (IS) shaft and its gears and bearings and the low speed (LS) shaft and its gear and bearings are from an ended implanted test case. Wear-in duration for the IS and LS shaft-related components lasted $10 \mathrm{~h}$ with different load and speed combinations.

Figure 7 illustrates a schematic of the experimental gearbox test rig. It includes a two-stage fixed-axis gearbox, a 4-kW three-phase asynchronous motor for driving the gearbox and a magnetic powder brake for loading. The motor speed can be adjusted for different values. The NI data acquisition system consists of four IEPE accelerometers (Dytran 3056B4-Dytran Instruments Inc., Fraser, MN, USA), PXI-1031 mainframe, PXI-4472B data acquisition cards and LabVIEW software (National Instrument Inc., Austin, TX, USA). In addition, a tachometer and torque sensor is installed in the input shaft for acquiring the speed and load information. The internal structure of the gearbox is depicted in Figure 7. The gearbox has three shafts supported by rolling element bearings. The LS shaft gear has 81 teeth and meshes with the IS shaft gear with 18 teeth. The IS shaft gear with 64 teeth meshes with the HS shaft with 35 teeth. Therefore, the overall gear ratio of the gearbox equals 8.22:1. In this case, study datasets with a $20-\mathrm{Hz}$ input shaft rate, a $20-\mathrm{kHz}$ sampling frequency, a $12-\mathrm{s}$ sampling duration, a 199-Nm and 405-Nm loads are used.

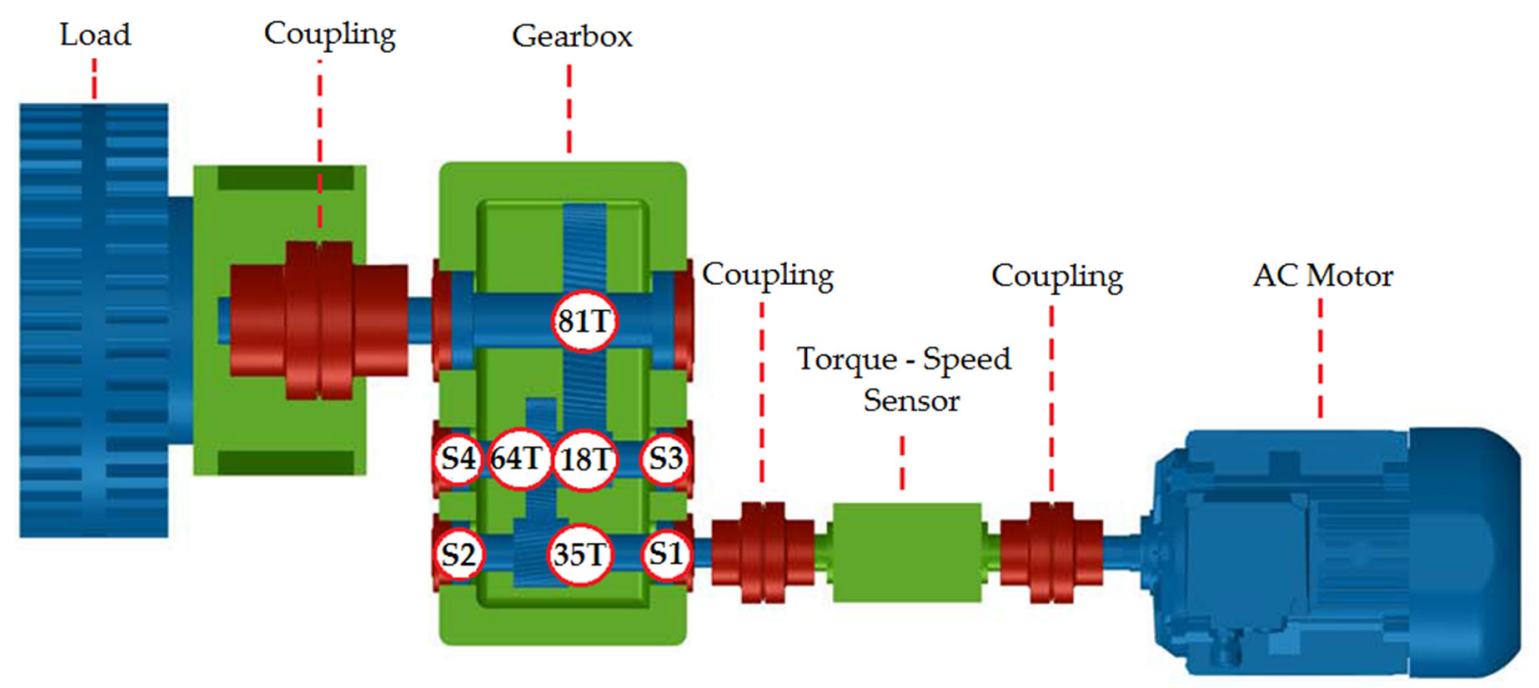

Figure 7. A schematic of the experimental gearbox test rig.

After the test, the gearbox was dismounted in order to check the bearings' condition. It was found that all bearings have faults at different levels. However, only the HS shaft and LS shaft bearings have obvious faults. Both HS shaft bearings had the outer race fault shown in Figure 8a,b, and the right bearing in addition had the inner race fault and ball fault shown in Figure 9a,b, respectively. Faults of the IS shaft bearing were not obvious faults. The left LS shaft bearing had the inner race fault and ball fault shown in Figure 10a,b, respectively. The HS and IS shaft are supported by SKF 6205 bearings and the LS shaft by SKF 6208 bearings. The fundamental faults frequencies of SKF 6205 under a 1-Hz rotating speed are BPFO $(3.585 \mathrm{~Hz})$, BPFI $(5.415 \mathrm{~Hz})$, BSF $(2.357 \mathrm{~Hz})$ and FTF $(0.398 \mathrm{~Hz})$. For SKF 6208, they are BPFO $(3.578 \mathrm{~Hz})$, BPFI $(5.423 \mathrm{~Hz})$, BSF $(2.337 \mathrm{~Hz})$ and FTF $(0.398 \mathrm{~Hz})$. Therefore, the actual fault frequencies can be acquired through these fundamental frequencies multiplied by the rotating frequency. The bearing types and the characteristic frequencies for a $20-\mathrm{Hz}$ input shaft rate are concerned and summarized in Table 1. 


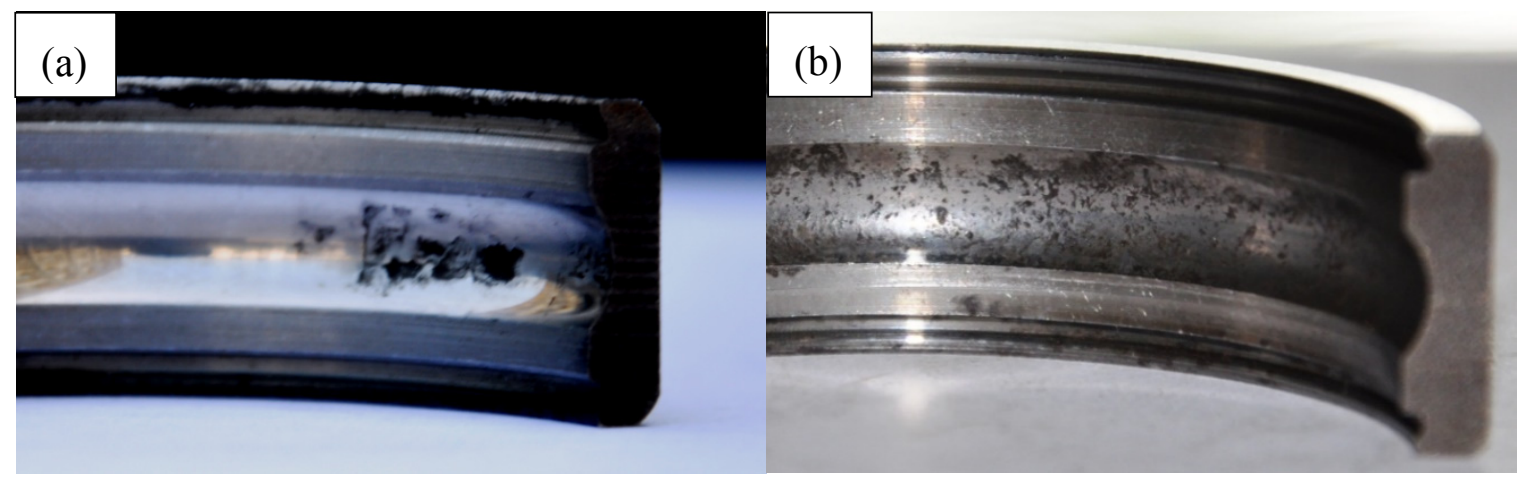

Figure 8. High speed (HS) shaft bearing outer race faults: (a) left bearing; (b) right bearing.

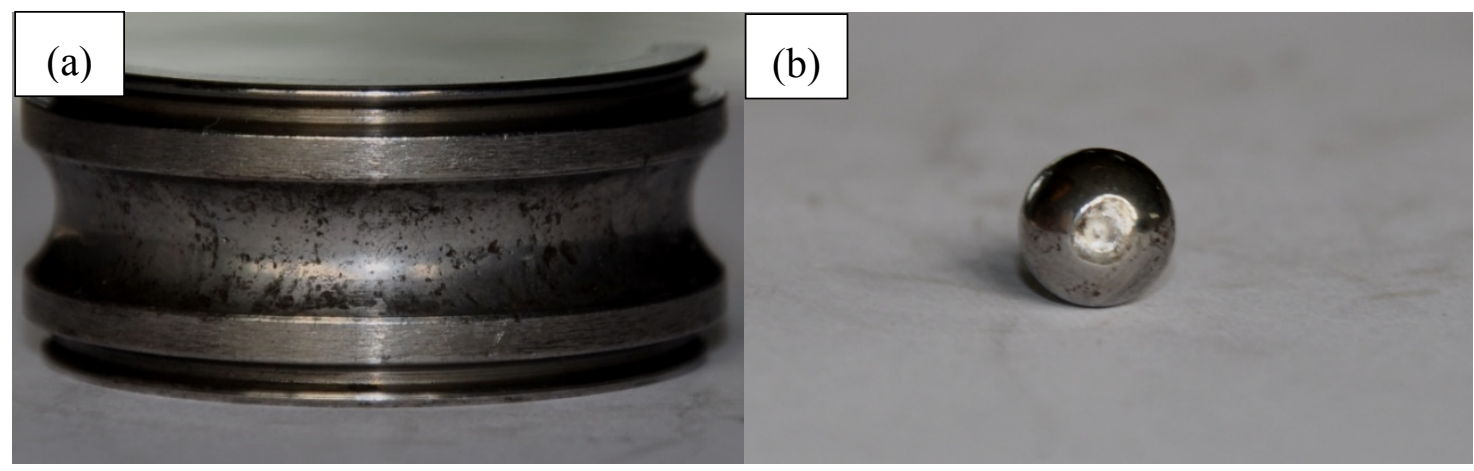

Figure 9. HS right bearing faults: (a) inner race fault; (b) ball fault.

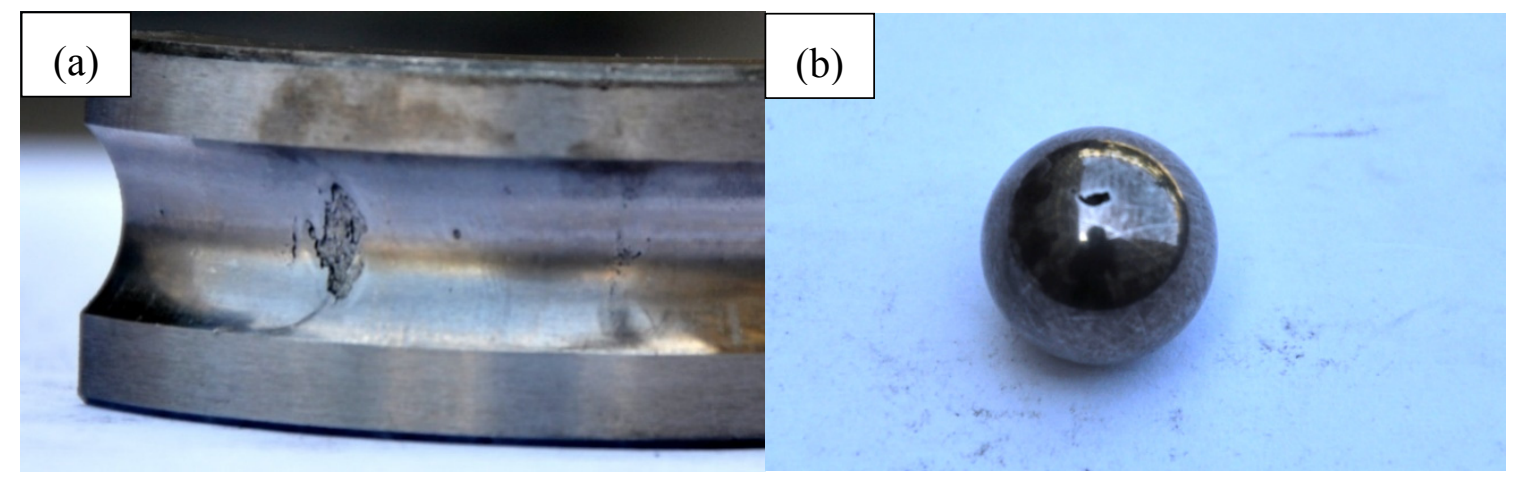

Figure 10. Low speed (LS) left bearing faults: (a) inner race fault; (b) ball fault.

Table 1. Characteristic frequencies of bearings (Hz). BPFO: ball pass frequency outer race; BPFI: ball pass frequency inner race; BSF: ball spin frequency; FTF: fundamental train frequency.

\begin{tabular}{ccccc}
\hline Bearing Type & BPFO & BPFI & BSF & FTF \\
\hline 6205 & 71.70 & 108.30 & 47.17 & 7.96 \\
6208 & 8.70 & 13.19 & 5.68 & 0.96 \\
\hline
\end{tabular}

It is very difficult to diagnose the bearing fault of the LS shaft because of the low speed and requirement of special techniques. In this case, only HS bearing faults under loads of $199 \mathrm{Nm}$ and $405 \mathrm{Nm}$ are considered. Except the bearing faults, the HS shaft gear with 35 teeth had a slight tooth wear fault. The IS shaft gear with 64 teeth had a chipped tooth fault, and the IS shaft gear with 18 teeth had a severe tooth wear fault shown in Figure 11. For a gear transmission with fixed axes, the gear 
meshing frequency (GMF) can be calculated by the following formula: GMF $=N \times Z$, where $N$ means the rotating speed of the test gear and $Z$ represents the number of the test teeth. According to this, the GMF of the HS and IS shaft gears is determined as $700 \mathrm{~Hz}$, and the GMF of the IS and LS shaft gears is determined as $196.93 \mathrm{~Hz}$.

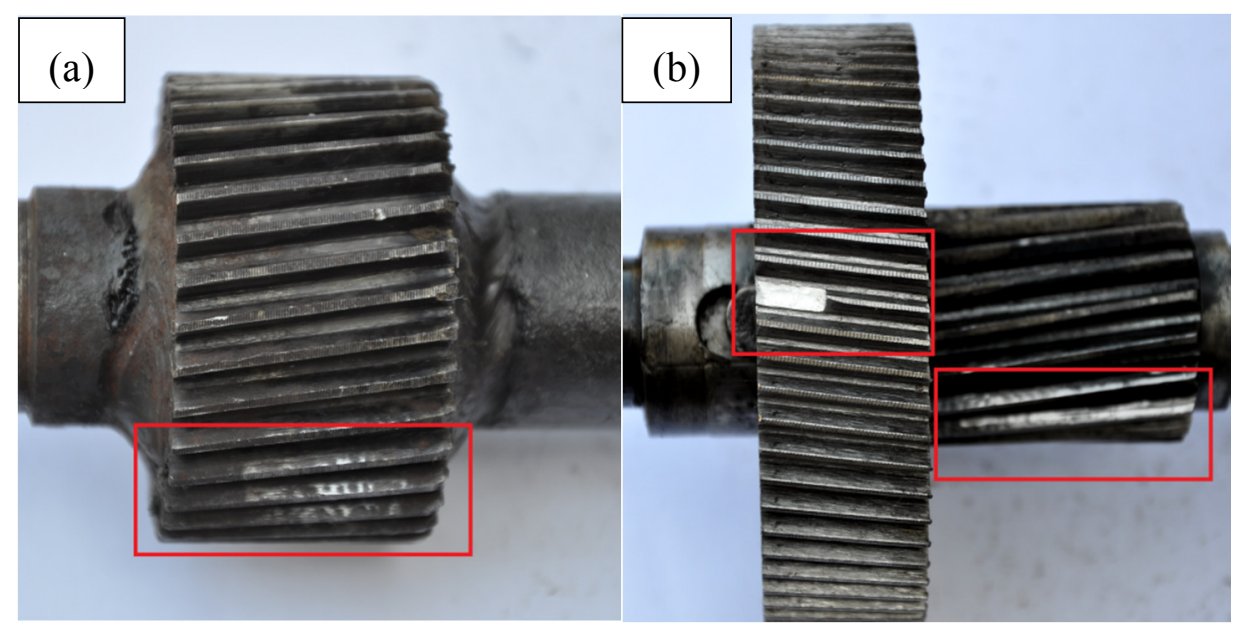

Figure 11. (a) HS shaft gear tooth wear; (b) intermediate speed (IS) shaft gears, tooth chip and wear.

First, in order to ensure stable signal, the data acquisition system is adjusted to use the data sample rate based on the tachometer signal. The adjusted sampling rate is a synchronous sampling rate and donates the resampled vibration signal. During the experiment, the load of $199 \mathrm{Nm}$ was applied to the output shaft (LS shaft), and the resampled signal is used. An accelerometer was mounted on the top of the gearbox casing (as shown in Figure 7), and the vibration signals were collected from the accelerometer at position S2. Figure 12a illustrates the correlation coefficient variation vs. the iteration number of DC offset cancellation, while Figure $12 \mathrm{~b}$ illustrates the CK value variation vs. the iteration number of DC offset cancellation. Figure 12c illustrates the squared envelope signal after the 100th iteration. It can be seen that both the correlation coefficient and CK cannot be used to determine the optimal iteration number for DC offset cancellation in the multi-fault mode case. Especially for the weak PIS, CK will not work. However, as mentioned in Section 2.1, one DC offset cancellation operation can subtract one harmonic deterministic component. In this case, HS shaft gear mesh frequency and its harmonics dominate the frequency spectrum, as shown in Figure 13. It can be seen that there are mainly five harmonics of the gear mesh frequency. Therefore, theoretically, only five-times DC offset cancellation can suppress the deterministic component of the original signal. Therefore, we conduct five iteration DC offset cancellation for the time synchronous signal. The squared envelope spectrum and its time-frequency contour are illustrated in Figure 14. After checking the squared envelope spectrum carefully, it is very hard to find the bearing fault frequencies. Instead of the bearing fault frequencies, the squared envelope spectrum is dominated by the HS and IS shaft rate and its harmonics because of the gear chip fault and wear fault. However, in the time-frequency contour of the squared envelope signal, weak BPFO, BPFI and BSF information can be seen. On the contrary, no bearing fault information from the envelope spectrum and time-frequency contour of the original signal shown in Figure 15 can be seen. Similarly, the envelope spectrum of the original signal is also dominated by the HS and IS shaft rate and its harmonics. 


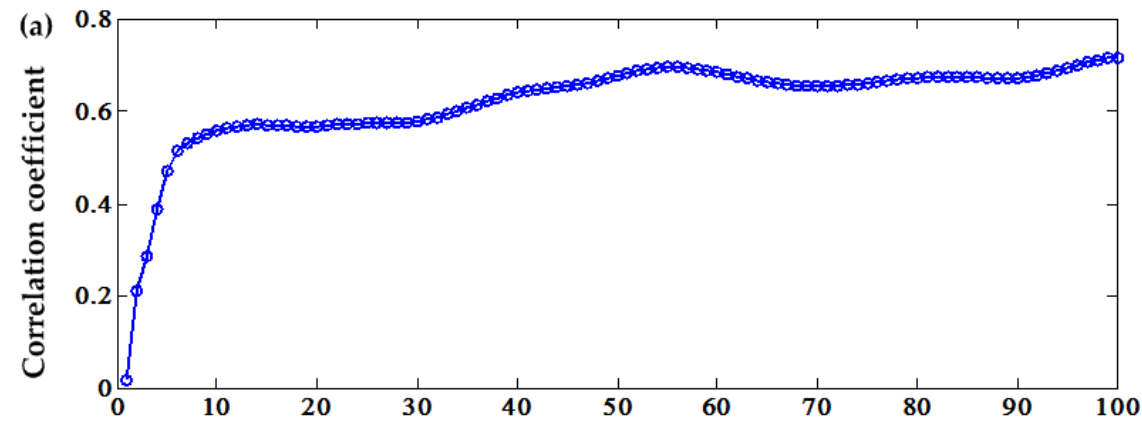

Iteration number
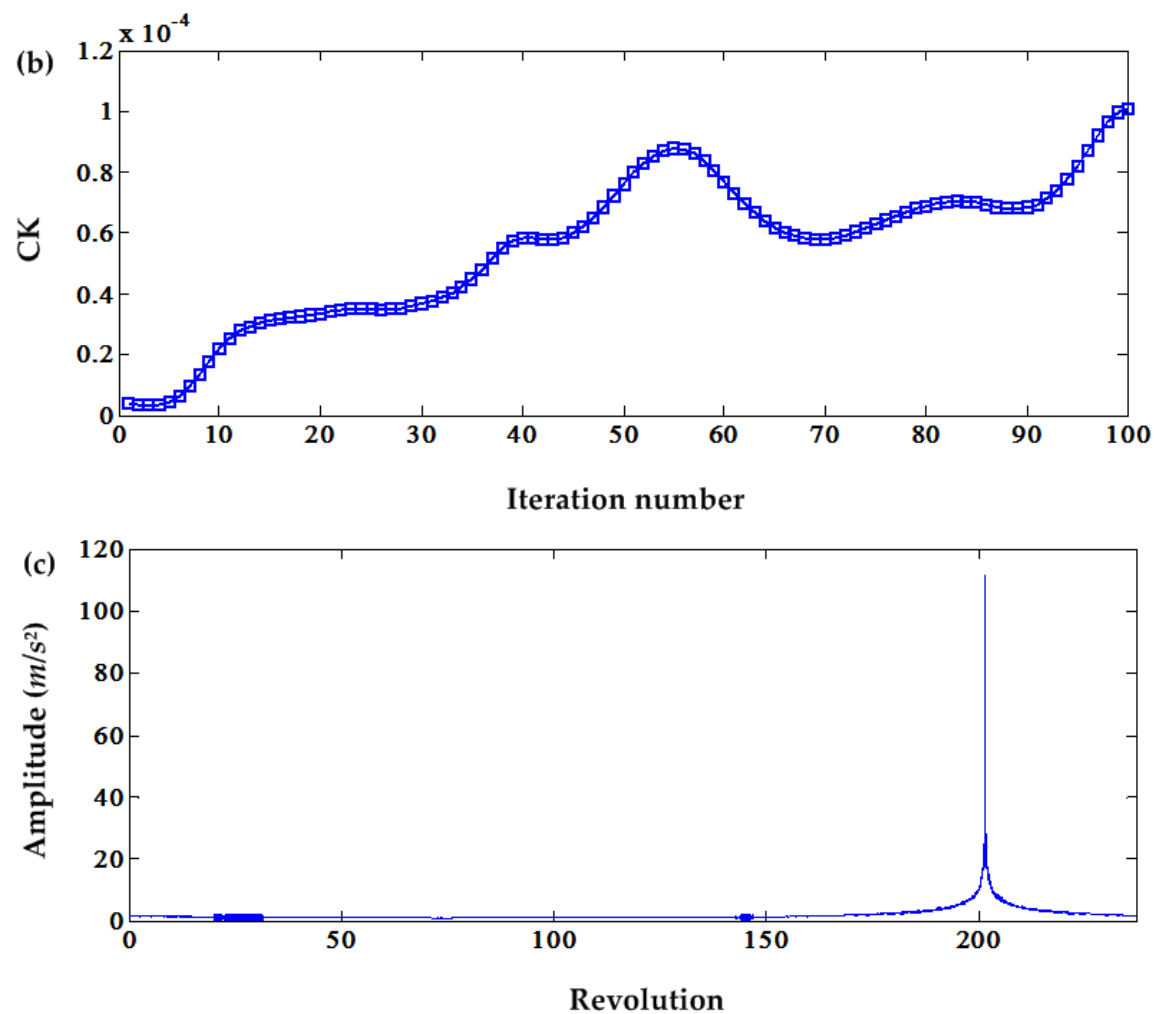

Figure 12. (a) Correlation coefficient vs. iteration number; (b) CK value vs. iteration number; (c) Squared envelope signal after the 100th DC offset cancellation.

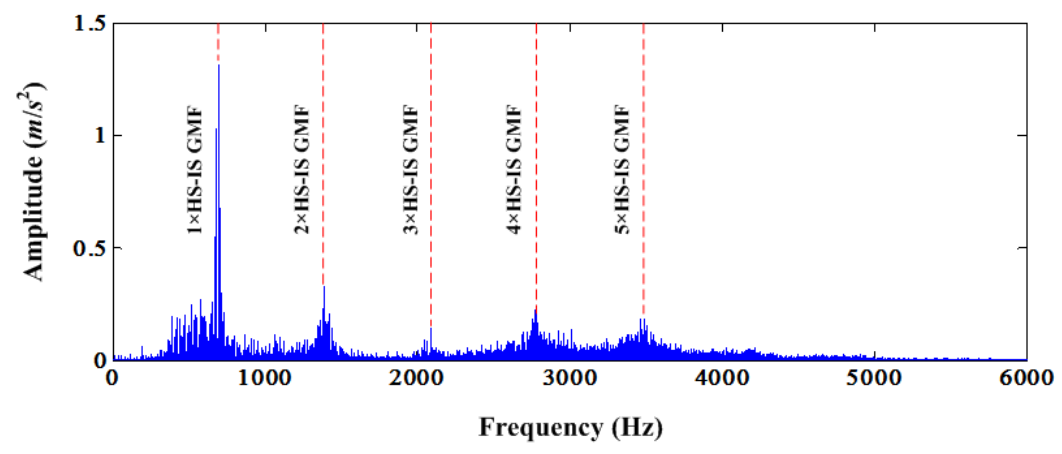

Figure 13. Frequency spectrum of the time synchronous signal; GMF, gear meshing frequency. 

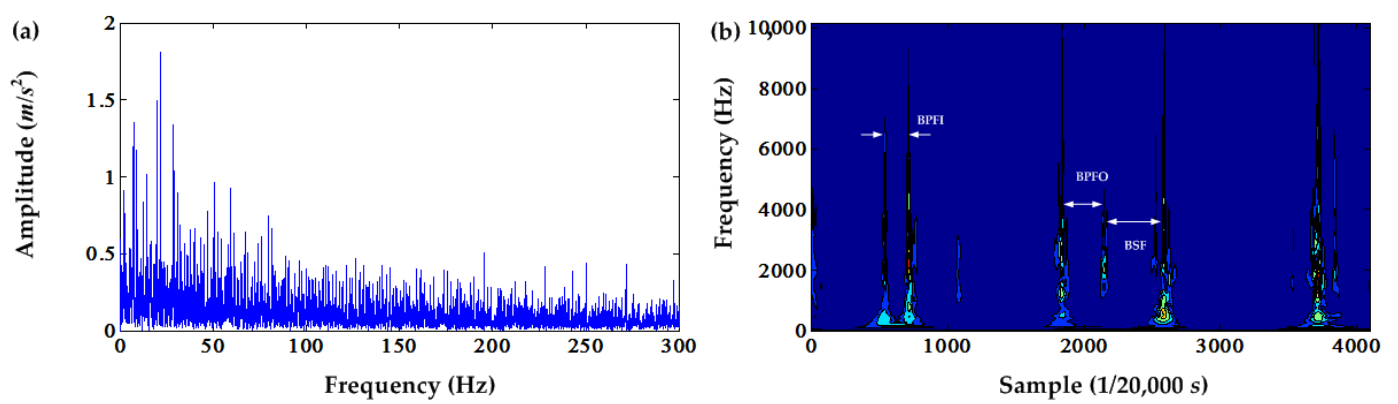

Figure 14. (a) Squared envelope spectrum after the fifth DC offset cancellation; (b) time-frequency contour of the squared envelope signal after the fifth DC offset cancellation.
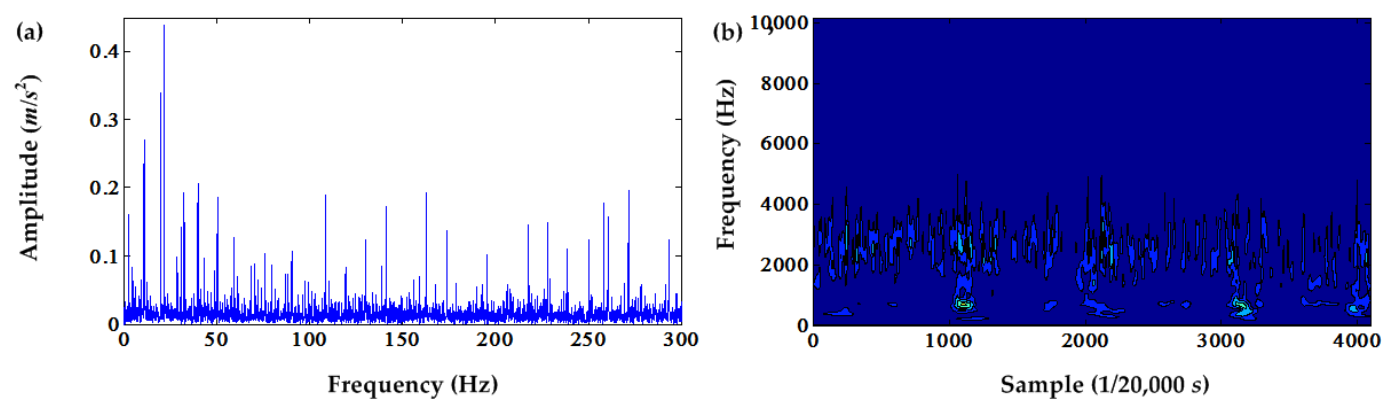

Figure 15. (a) Envelope spectrum of the original signal; (b) time-frequency contour of the original signal.

Second, in order to ensure a stable signal, the data acquisition system is adjusted to use the data sample rate based on the tachometer signal. The adjusted sampling rate is a synchronous sampling rate and donates the resampled vibration signal. During the experiment, the load of $405 \mathrm{Nm}$ was applied to the output shaft (LS shaft), and resampled signal is used. An accelerometer was mounted on the top of the gearbox casing (as shown in Figure 7), and the vibration signals were collected from the accelerometer at position S2. Figure 16 illustrates the frequency spectrum of the time synchronous signal with the dominant six harmonics of the HS-IS GMF. Therefore, for the DC offset cancellation operation, we can repeat six times to delete the deterministic component. Finally, the squared envelope spectrum and relevant time-frequency contour can be acquired as shown in Figure 17. Similarly, the squared envelope spectrum is dominated by the HS and IS shaft rate and its harmonics. Information about the bearing fault frequencies cannot be found in the squared envelope spectrum. However, weak BPFI and BSF can be found in the time-frequency contour by the $S$ transform. In order to compare with the traditional envelope analysis, the envelope spectrum of the time synchronous signal is given in Figure 18a. The time-frequency contour of the envelope signal of the time synchronous signal is illustrated in Figure 18b. It can be seen that it is very difficult to find any bearing fault-related information from this time-frequency contour.

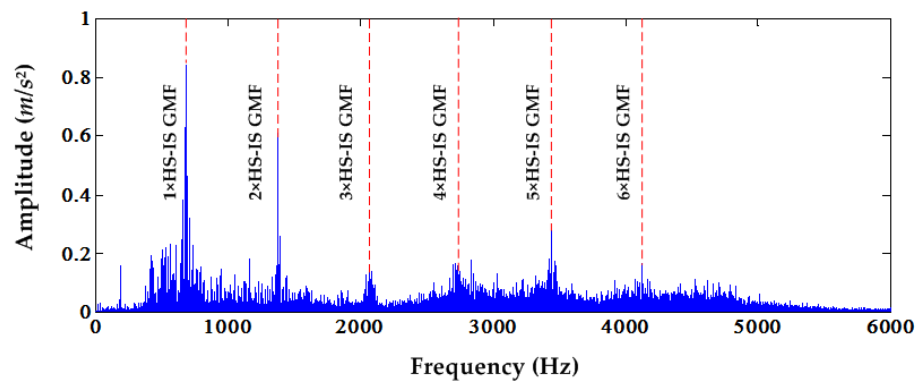

Figure 16. Frequency spectrum of the time synchronous signal. 

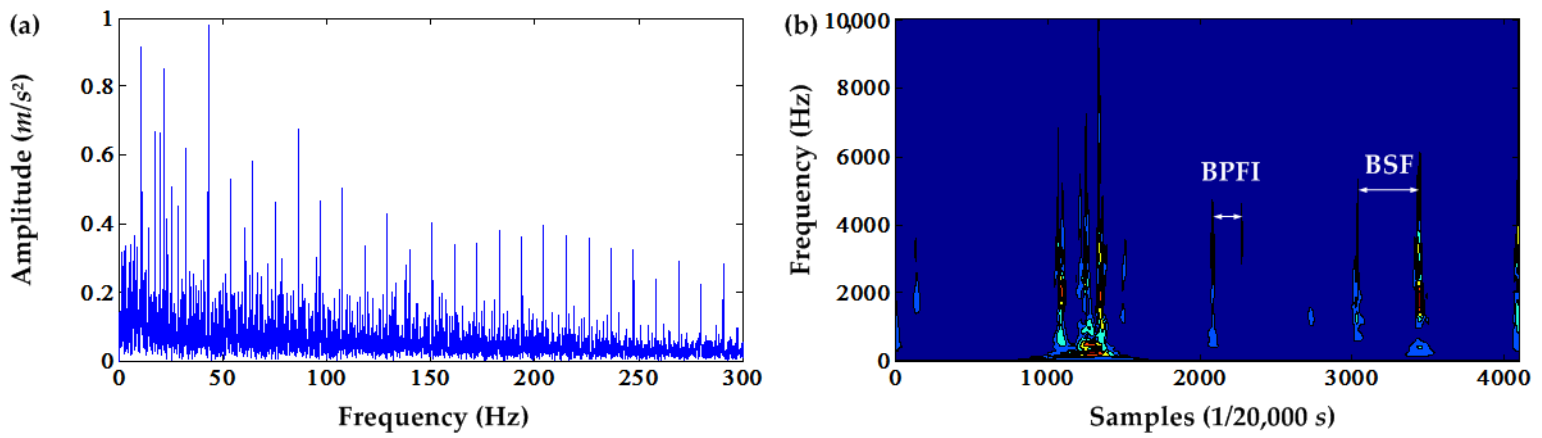

Figure 17. (a) Squared envelope spectrum after the sixth DC offset cancellation; (b) time-frequency contour of the squared envelope signal after the sixth DC offset cancellation.
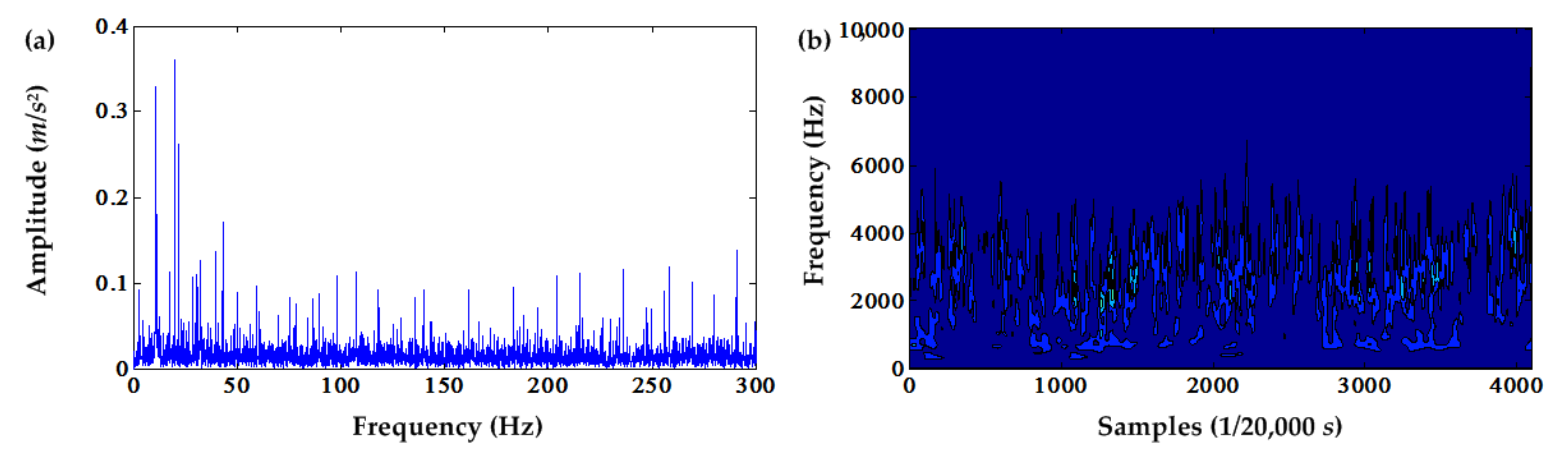

Figure 18. (a) Envelope spectrum of the time synchronous signal; (b) time-frequency contour of the envelope signal of the original signal.

\section{Novel Proposed Method Application for Planetary Gearbox Fault Diagnosis}

In this section, for the novel method application, experimental signals are collected from a lab planetary gearbox test rig with implanted wear tooth faults on the sun, planet and ring gear. Figure 19 illustrates a schematic of the experimental planetary gearbox test rig. It includes a single-stage planetary gearbox, a 4-kW three-phase asynchronous motor for driving the gearbox and a magnetic powder brake for loading. The motor speed can be adjusted for different values. The load can be adjusted by a brake controller through the current of the magnetic powder brake connected to the output shaft (carrier). The NI data acquisition system consists of four IEPE accelerometers (Dytran 3056B4, Dytran Instruments Inc., Fraser, MN, USA), PXI-1031 mainframe, PXI-4472B data acquisition cards and LabVIEW software (National Instrument Inc., Austin, TX, USA). In addition, a tachometer and torque sensor is installed in the input shaft for acquiring the speed and load information. The single-stage planetary gearbox consists of the sun gear, planet gear and fixed ring gear. The sun gear is connected to the input shaft and rotates around its own center. All planet gears mesh simultaneously with the sun gear and ring gear. These planet gears not only rotate around their own centers, but also revolve around the center of the sun gear, and vibration signals picked up by a fixed sensor attached to the planetary gearbox housing differ significantly from that of fixed-axis/parallel gear systems [32]. Compared to the fixed-axis gearbox test rig in Section 4, all of the settings are the same, except the gearbox type. Single-stage planetary gearbox gear parameters are listed in Table 2.

Table 2. Gear parameters of the single-stage planetary gearbox.

\begin{tabular}{ccccc}
\hline Gear & Sun & Planet & Ring & Planet Number \\
\hline Teeth number & 13 & 64 & 146 & 3 \\
\hline
\end{tabular}




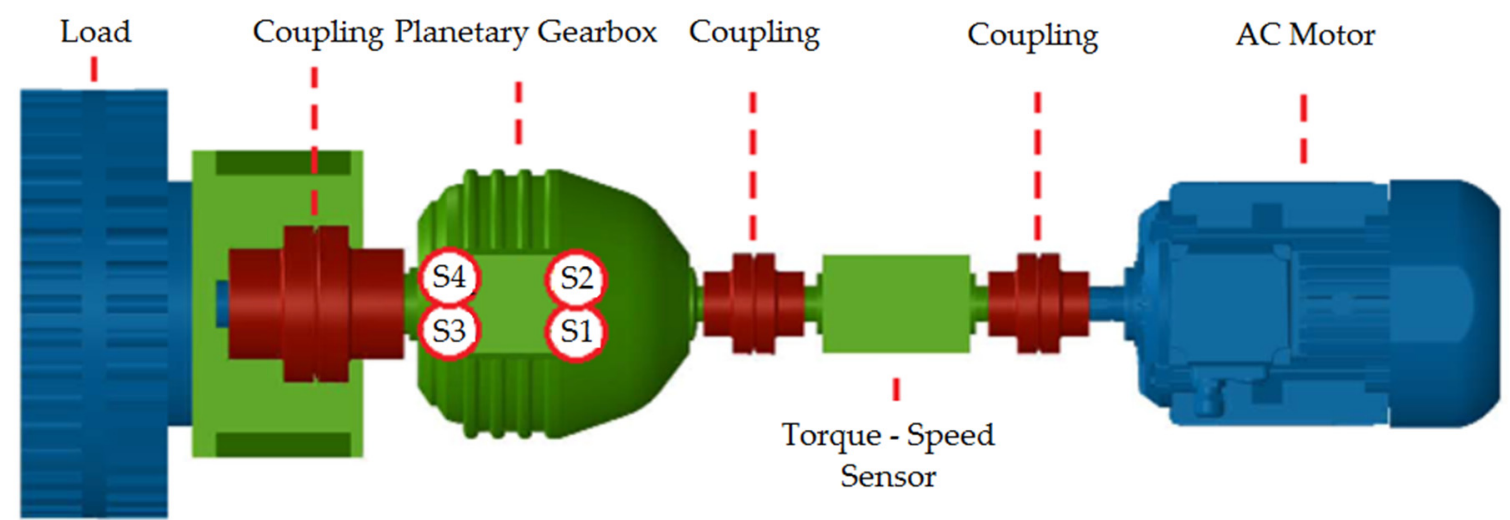

Figure 19. A schematic of the experimental planetary gearbox test rig.

Wear gear faults of the planetary gearbox were implanted in one tooth of the ring gear, planet gear and sun gear, respectively, in order to validate the proposed analysis method. Figure 20 illustrates the implanted gear faults.

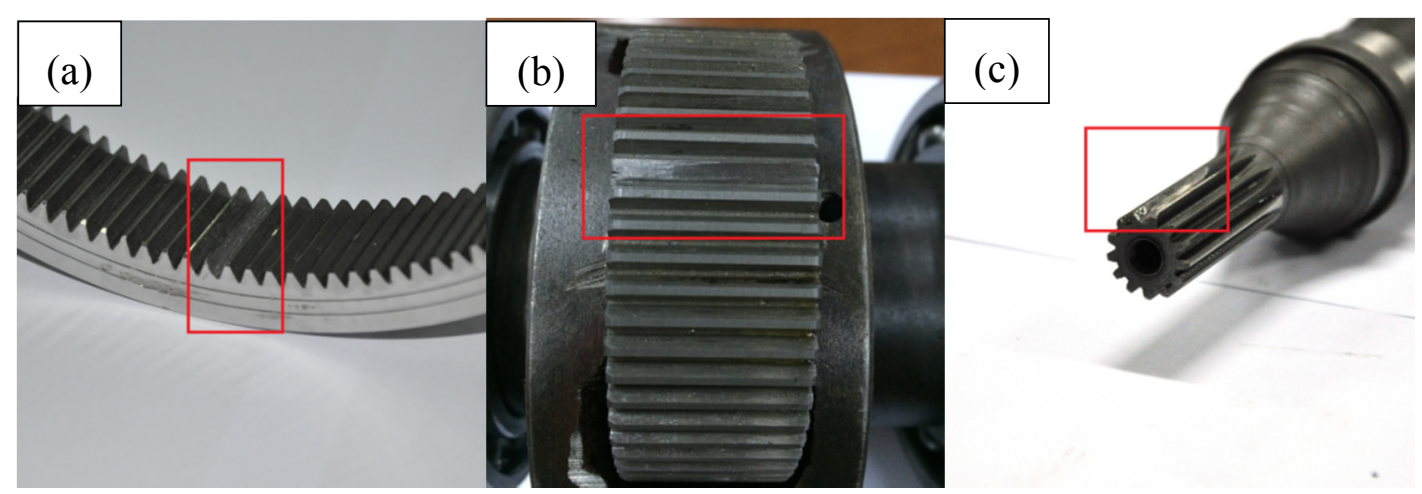

Figure 20. Implanted wear fault: (a) ring gear; (b) planet gear; (c) sun gear.

Tooth wear faults belong to localized fault, and their fault frequencies can be calculated through the following equations [33]. The meshing frequency for the stationary ring gear $f_{\text {mesh }}=f_{\text {carrier }} \times$ $Z_{\text {ring }}$ equals the product of the planet carrier rotating frequency $f_{\text {carrier }}$ and the number of ring gear teeth $Z_{\text {ring }}$. Relative rotating frequency with respect to the planet carrier is $f_{\text {relative }}=f_{\text {mesh }} / Z$, where $Z$ is the total number of teeth of the gear of interest. The characteristic frequency of faulty planet gear is $f_{\text {planet1 }}=f_{\text {mesh }} / Z_{\text {planet }}$, where $Z_{\text {planet }}$ is the number of planet gear teeth. If the damage exists on both sides of the gear tooth, the damaged planet gear tooth also meshes with the other mating gear. The characteristic frequency of the faulty planet gear becomes $f_{\text {planet2 }}=2 \times f_{\text {mesh }} / Z_{\text {planet }}$. The characteristic frequency of the faulty sun gear equals the relative rotating frequency of the sun gear multiplied by the number of planet gears $f_{\text {sun }}=N_{\text {planet }} \times f_{\text {mesh }} / Z_{\text {sun }}$, where $Z_{\text {sun }}$ is the number of sun gear teeth and $N_{\text {planet }}$ is the number of planet gears. The characteristic frequency of the faulty ring gear is $f_{\text {ring }}=N_{\text {planet }} \times f_{\text {mesh }} / Z_{\text {ring }}$. Similarly, for the distributed damage case, the characteristic frequency of the faulty ring gear is $f_{\text {ring }}=f_{\text {mesh }} / Z_{\text {ring }}$.

\subsection{Sun Gear Fault Experiment}

For this experiment, we used a sun gear wear fault mode to test the proposed method while the remaining gears were all in good condition, undamaged. Similar to the case study in Section 4, in order to ensure a stable signal, the data acquisition system is adjusted to use the data sample rate based on the tachometer signal. The adjusted sampling rate is a synchronous sampling rate and donates the resampled vibration signal. During the experiment, the mean rotating frequency of the 
input shaft connecting the sun gear of the planetary gearbox was $20.0889 \mathrm{~Hz}$, while a load of $405 \mathrm{Nm}$ was applied to the output shaft connecting the planet carrier. An accelerometer was mounted on the top of the gearbox casing (as shown in Figure 19), and the vibration signals were collected from the accelerometer at position S3. According to the planetary gearbox configuration and its running speed, the characteristic frequencies are calculated and listed in Table 3.

Table 3. Characteristic frequencies (Hz).

\begin{tabular}{ccccccc}
\hline \multirow{2}{*}{ Meshing Frequency } & \multicolumn{2}{c}{ Rotating Frequency } & \multicolumn{4}{c}{ Local Damage } \\
\cline { 2 - 7 } & Sun & Carrier & \multicolumn{2}{c}{ Planet } & Sun & Ring \\
\hline 239.8183 & 20.0889 & 1.6425 & 3.7472 & 7.4944 & 55.3427 & 4.9278 \\
\hline
\end{tabular}

According to the framework of the proposed method, the squared envelope signal after DC offset cancellation is acquired. For this case, the CK value shows that the first envelope has the biggest PIS, as shown in Figure 21. The squared envelope spectrum after the first DC offset cancellation and the envelope spectrum of only the time synchronous signal are illustrated in Figure 22. Compared to the envelope spectrum of the original signal, the amplitudes of the sun gear fault frequency and its harmonics are much higher compared to the envelope spectrum of the original signal. However, it can also be noticed that the noise amplitudes unrelated to the sun gear fault frequency are also higher compared to the envelope spectrum of the original signal.

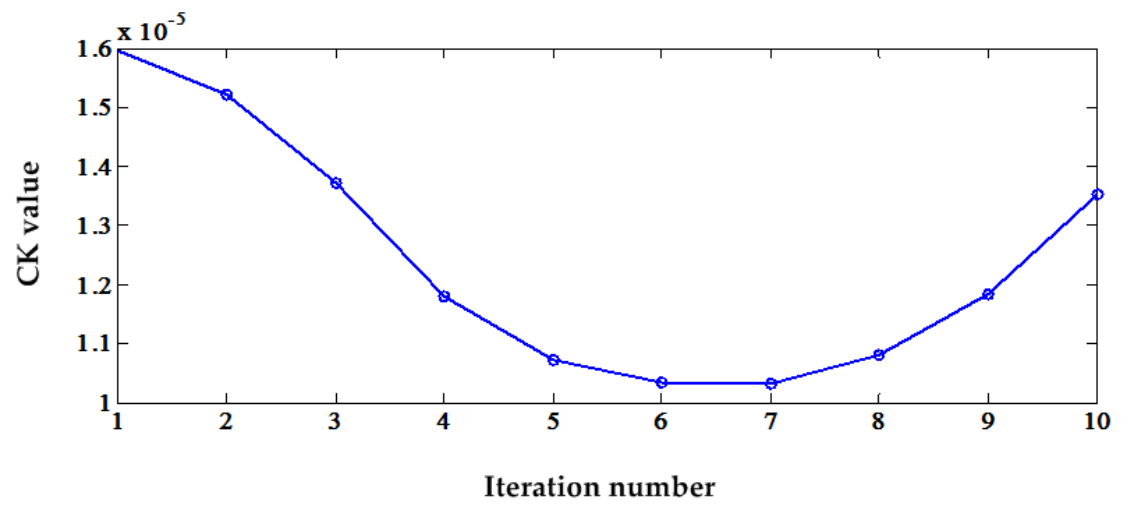

Figure 21. CK value variation vs. iteration number.
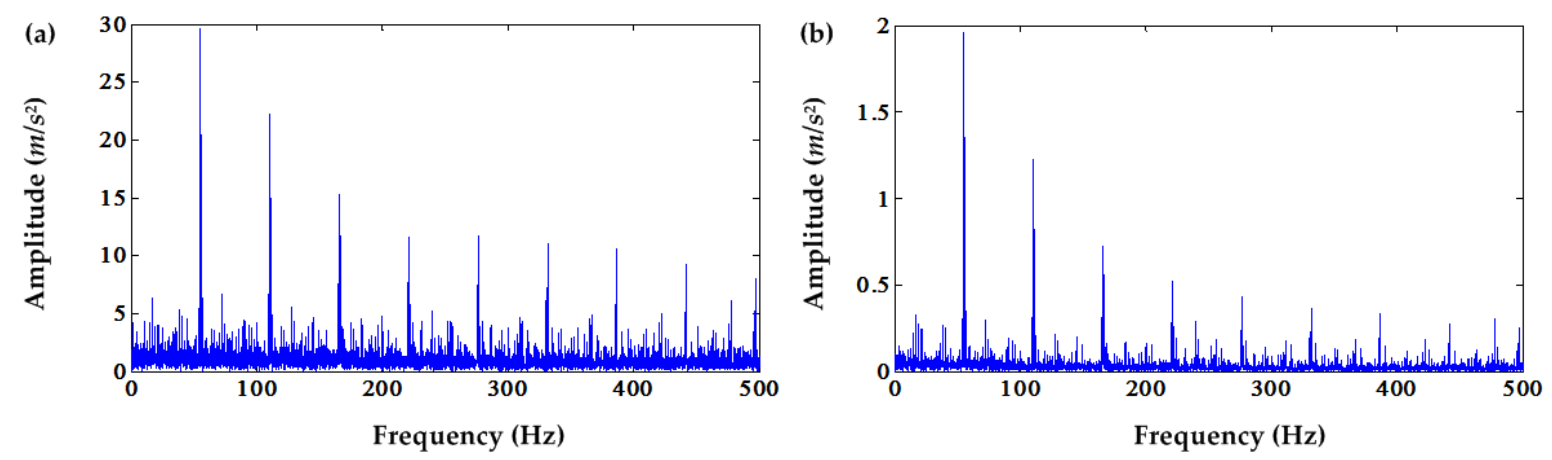

Figure 22. (a) Squared envelope spectrum after the first DC offset cancellation; (b) envelope spectrum of the original time synchronous signal.

The time-frequency contour of the squared envelope signal using the $\mathrm{S}$ transform is shown in Figure 23. In contrast, the time-frequency contour of the original envelope signal using the $S$ 
transform is shown in Figure 24. From these two figures and their local amplifications, the sun gear fault frequency in the frequency domain and the time-frequency domain can be seen. However, the time-frequency contour of the squared envelope signal has less useful sun gear fault information than the time-frequency contour of the original envelope signal.
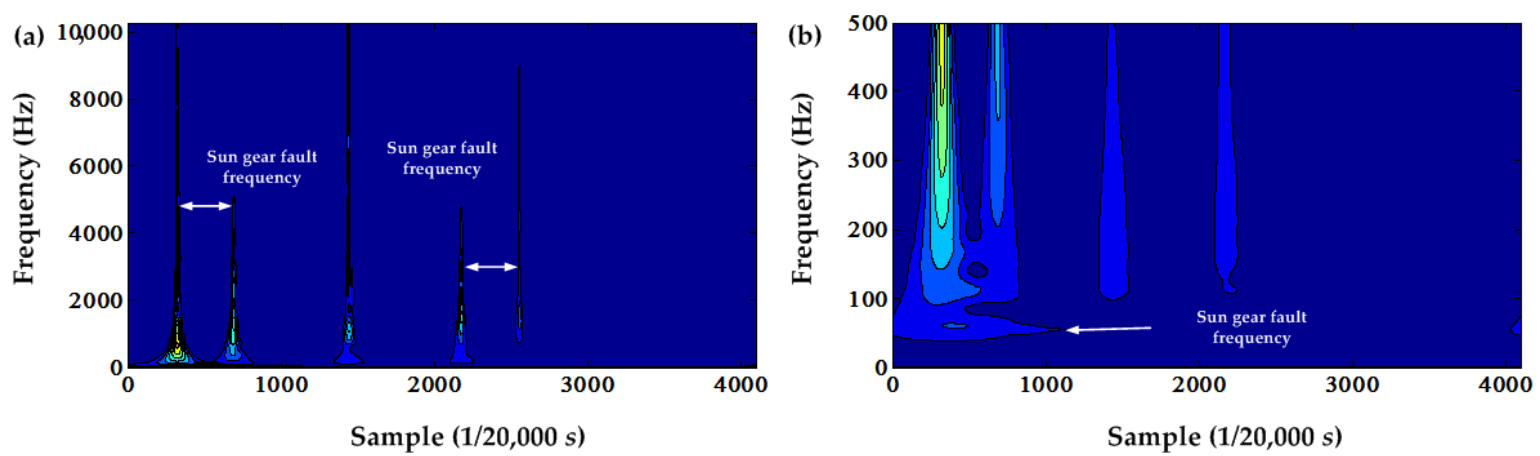

Figure 23. (a) Time-frequency contour of the squared envelope signal using the $S$ transform; (b) local amplification of (a).
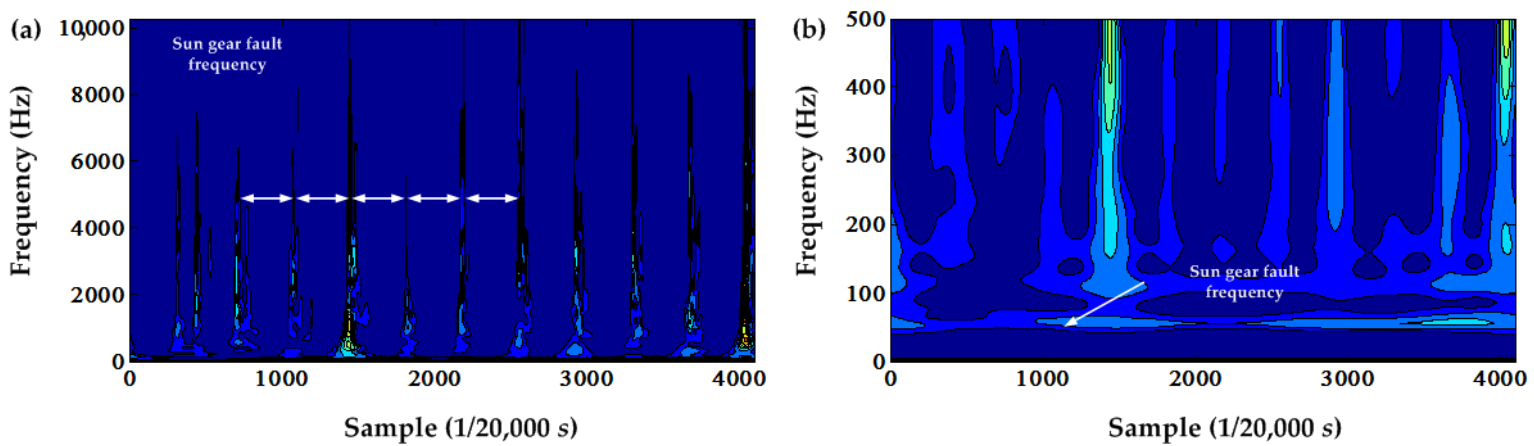

Figure 24. (a) Time-frequency contour of the original envelope signal using the $S$ transform; (b) local amplification of (a).

\subsection{Planet Gear Fault Experiment}

For this experiment, we analyzed one planet gear wear fault mode to test the proposed method while the remaining gears were all in good condition, undamaged. Similar to the case study in Section 4, in order to ensure a stable signal, the data acquisition system is adjusted to use the data sample rate based on the tachometer signal. The adjusted sampling rate is a synchronous sampling rate and donates the resampled vibration signal. During the experiment, the mean rotating frequency of the input shaft connecting the sun gear of the planetary gearbox was $20.0111 \mathrm{~Hz}$, while a load of $405 \mathrm{Nm}$ was applied to the output shaft connecting the planet carrier. An accelerometer was mounted on the top of the gearbox casing (as shown in Figure 19), and the vibration signals were collected from the accelerometer at position S3. According to the planetary gearbox configuration and its running speed, the characteristic frequencies are calculated and listed in Table 4.

Table 4. Characteristic frequencies $(\mathrm{Hz})$.

\begin{tabular}{ccccccc}
\hline \multirow{2}{*}{ Meshing Frequency } & \multicolumn{2}{c}{ Rotating Frequency } & \multicolumn{4}{c}{ Local Damage } \\
\cline { 2 - 7 } & Sun & Carrier & \multicolumn{2}{c}{ Planet } & Sun & Ring \\
\hline 238.8748 & 20.0111 & 1.6361 & 3.7324 & 7.4648 & 55.1249 & 4.9083 \\
\hline
\end{tabular}


Similarly, we can apply the DC offset cancellation operation to this signal. The CK value shows that the signal only after the first DC offset cancellation iteration has the strongest PIS. The CK value variation vs. the iteration number can be seen in Figure 25. Though the CK achieves highest value at the 10th iteration, after Iteration 6, the envelope signal is corrupted seriously. Therefore, the first iteration is optimal. The comparison of the squared envelope spectrum and original envelope spectrum is shown in Figure 26. It can be seen that there is no difference in essence, except the amplitude. Because of the very small planet gear fault frequencies, it is very difficult to display the time-frequency contour using the $\mathrm{S}$ transform.

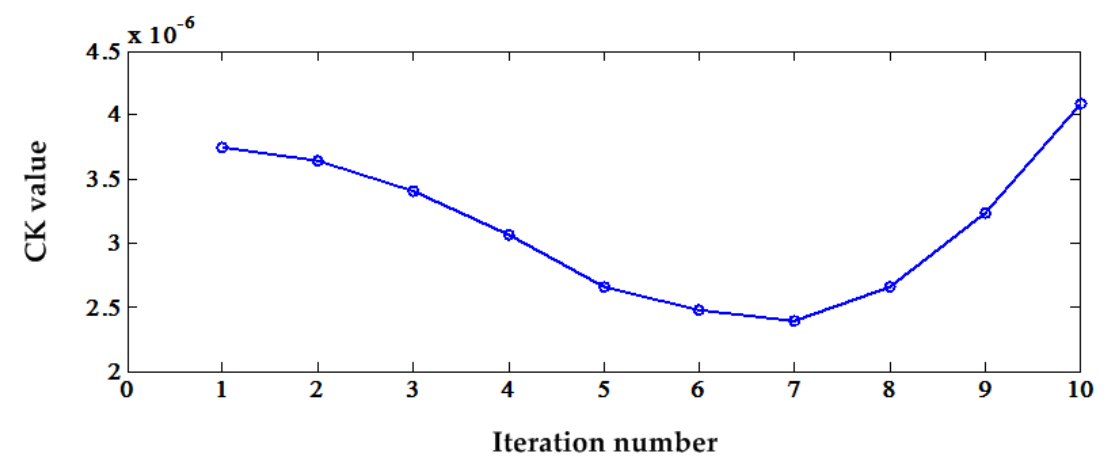

Figure 25. CK value variation vs. iteration number.
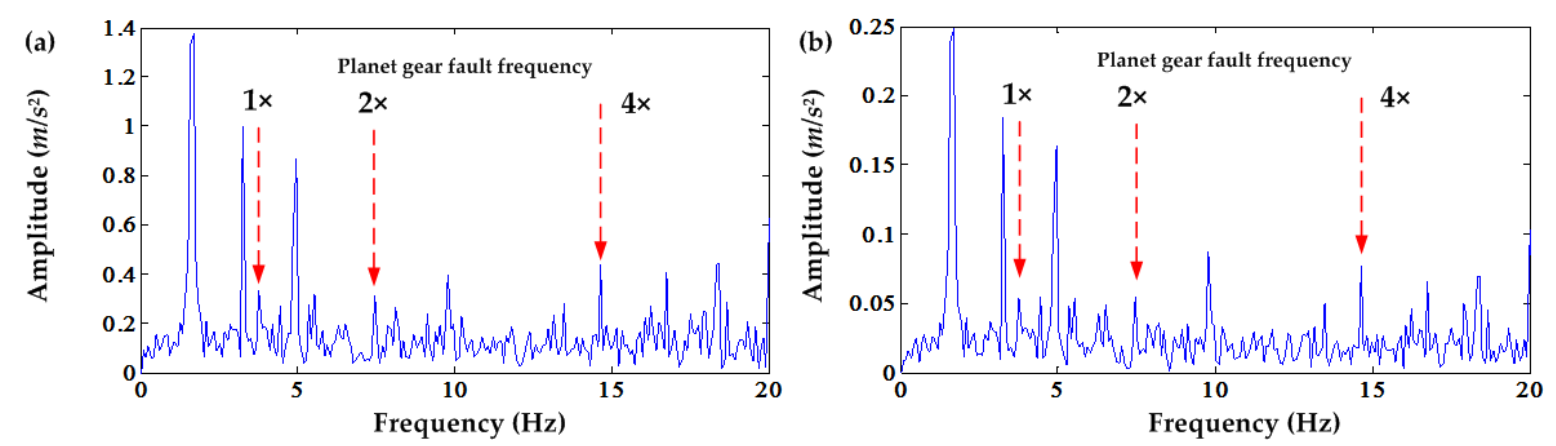

Figure 26. (a) Squared envelope spectrum after the first DC offset cancellation; (b) envelope spectrum of the original signal.

\subsection{Ring Gear Fault Experiment}

For this experiment, we used a ring gear wear fault mode to test the proposed method while the remaining gears were all in good condition, undamaged. Similar to the case study in Section 4 , in order to ensure a stable signal, the data acquisition system is adjusted to use the data sample rate based on the tachometer signal. The adjusted sampling rate is a synchronous sampling rate and donates the resampled vibration signal. During the experiment, the mean rotating frequency of the input shaft connecting the sun gear of the planetary gearbox was $22.3889 \mathrm{~Hz}$, while a load of $405 \mathrm{Nm}$ was applied to the output shaft connecting the planet carrier. An accelerometer was mounted on the top of the gearbox casing (as shown in Figure 19), and the vibration signals were collected from the accelerometer at position S3. According to the planetary gearbox configuration and its running speed, the characteristic frequencies are calculated and listed in Table 5. 
Table 5. Characteristic frequencies $(\mathrm{Hz})$.

\begin{tabular}{ccccccc}
\hline \multirow{2}{*}{ Meshing Frequency } & \multicolumn{2}{c}{ Rotating Frequency } & \multicolumn{4}{c}{ Local Damage } \\
\cline { 2 - 7 } & Sun & Carrier & \multicolumn{2}{c}{ Planet } & Sun & Ring \\
\hline 267.2586 & 22.3889 & 1.8305 & 4.1759 & 8.3518 & 61.6751 & 5.4916 \\
\hline
\end{tabular}

The ring gear fault frequency is not always calculated in the same way. Theoretically, its fault frequency is $f_{\text {mesh }}$. However, because of manufacturing error, the three planet gears are different from each other. Therefore, the ring gear fault frequency may be $1 / 3 f_{\text {mesh }}$. Therefore, it is very difficult to determine the parameter $T$ in CK. In this case, we can continue to apply the first DC offset cancellation to the signal. The resulting squared envelope spectrum and the original signal envelope spectrum can be seen in Figure 27. Similarly, it is possible to find the second order ring gear fault frequency in both envelope spectrums. There is no difference, except the value of the amplitude.
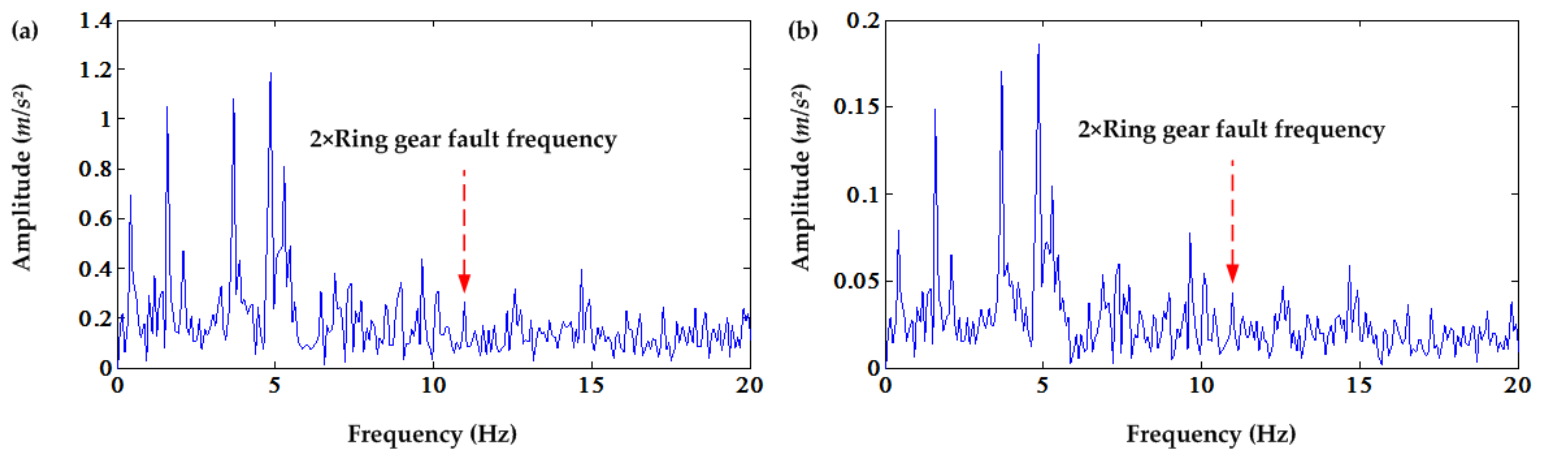

Figure 27. (a) Squared envelope spectrum after the first DC offset cancellation; (b) the envelope spectrum of the original signal.

\section{Discussion}

In this paper, three experimental case studies are conducted for the novel deterministic component cancellation method application in detail. Not only amplitude-frequency characteristics, but also the time-frequency characteristics are analyzed by the $S$ transform. The methods' application in those experimental case studies reveals issues that need to be further discussed and investigated:

(1) The cross-correlation coefficient indicator should be investigated more. The region $[0.9,0.95]$ is not appropriate for the illustrated cases in this paper. Therefore, there is a need for further study in order to determine the optimal iteration number and a more robust region.

(2) The CK indicator takes advantage of when the PIS are more powerful than the other signal components. The case study in Section 3 validates the implanted bearing outer race fault diagnosis, with only bearing fault mode present, and confirms the powerful PIS characteristic of implanted faults. It is confirmed that the CK gives excellent results in determining the optimal iteration number of DC offset cancellation. However, when corrupted by the excess DC offset cancellation operation, the envelope signal analysis will not give a good result because the PIS are also corrupted. For the case study in Section 4, all of the bearing faults are naturally developed. Simultaneously, there are gear chip faults and wear faults. Therefore, the PIS produced by these bearing faults are very weak. In this case, the CK gives a poor result. Theoretically, every DC offset cancellation can suppress one harmonic of the deterministic component of the original signal. For the fixed-axis gearbox, the deterministic component is mainly the HS-IS gear mesh frequency and its harmonics. This is illustrated in Figures 13 and 16 in Section 4. Therefore, it is possible to determine the optimal iteration number by the number of HS-IS gear mesh harmonics. This is very useful for a fixed-axis gearbox. Whereas there is another problem, no 
matter if the squared envelope spectrum after DC offset cancellation or the envelope spectrum of originals signal is used, they are both dominated by the shaft rate and harmonics related to the HS and IS shaft. No information about bearing fault frequencies can be found. Fortunately, the time-frequency contour of the squared envelope signal after DC offset cancellation by the $S$ transform can reveal some information about the bearing fault frequencies. This demonstrates the iterative DC offset cancellation's effectiveness for PIS enhancement. For the implanted bearing outer race fault case study in Section 3, even though both the squared envelope spectrum after DC offset cancellation and the envelope spectrum of the original signal can reveal the bearing fault information effectively, however, the time-frequency contour of the squared envelope signal after DC offset cancellation has good time-frequency characteristics for revealing the bearing fault information, in comparison to the case without DC offset cancellation.

(3) Concerning planetary gearbox fault diagnosis, the cross-correlation coefficient has a similar problem as the previous case studies. The CK has the correct assessment for the iteration number for the sun gear fault and the planet gear fault. However, the CK does not take advantage of the ring gear fault, because of the uncertainty for the fault frequency. Besides the iteration number determined by the $\mathrm{CK}$ value, several tests for the iteration number between one and ten show that only the first DC offset cancellation gives the best performance. In addition, the mesh frequency always has high order harmonics greater than 10 for these data, and the number of harmonics cannot be used to determine the optimal iteration number. The squared envelope spectrums after the first DC offset cancellation do not have obvious superiority with respect to the original envelope analysis for the spectrum's efficient evaluation. This may be due to the different structures of the planetary and fixed-axis gearbox. The GMF of the fixed-axis gearbox is related to the rotating frequency of the gear and the gear teeth; however, for the planetary gearbox, the GMF depends on the gearbox rotating frequency and its configuration, which is more complex than the configuration of the fixed-axis gearbox. Therefore, how to revise the DC offset cancellation method to fit the planetary gearbox fault diagnosis is a problem which needs further investigation and explorations.

(4) The time-frequency contour by the $\mathrm{S}$ transform needs more computational capacity. Therefore, only short time-frequency characteristics can be displayed. However, for the planet gear fault and the ring gear fault, their fault frequencies are very small. This will lead to the large space between the time-frequency lines. Usually, it is not available to display the characteristics of the small fault frequencies. This problem also needs further investigation and explorations.

\section{Conclusions}

In order to detect the PIS signal produced by the gearbox fault, DC offset cancellation was used to process the fault signal. Through a comparative study based on an implanted bearing fault case, the $\mathrm{CK}$ was demonstrated to be superior to the cross-correlation coefficient to determine the iteration number of DC offset cancellation. For the implanted bearing fault case, the PIS produced by the fault are very strong. Therefore, detection of the PIS using CK is more effective. However, for the naturally-developed bearing fault compound with gear fault, the PIS are very weak for the CK to detect. In this circumstance, the CK loses its power and cannot find the proper iteration number for DC offset cancellation. The order of the high-speed shaft gear mesh frequency was used as the iteration number because every DC offset cancellation can suppress one harmonic mesh frequency. This is very useful for PIS extraction. All of above results are demonstrated on a fixed-axis gearbox. For a planetary gearbox, DC offset cancellation has a limited effect on the fault diagnosis enhancement, especially for those low fault frequency parts, such as the planet gear, ring gear, etc. In this paper, because of the merits, the $\mathrm{S}$ transform was used to display the time-frequency characteristics of the DC offset results. This made us observe the fault information from the time-frequency contour which is more intuitive. However, for the low fault frequency parts, it is very difficult for the time-frequency contour to display them because of the long samples. At the end of this paper, we had a detailed discussion about the DC 
offset cancellation and the $\mathrm{S}$ transform-based application. In the future, these time-frequency contours will be used for deep learning-based fault classification.

Acknowledgments: The research is partially supported by the China NSFC under Grant Number 71401173 and by the Natural Science Foundation of Hebei Province under Grant Number E2015506012.

Author Contributions: J.Z. and L.W. conceived of and designed the experiments. X.Z. performed the experiments. X.Z. and R.B. analyzed the data. X.Z. wrote the paper.

Conflicts of Interest: The authors declare no conflict of interest.

\section{References}

1. Jardine, A.K.S.; Lin, D.; Banjevic, D. A review on machinery diagnostics and prognostics implementing condition-based maintenance. Mech. Syst. Signal Process. 2006, 20, 1483-1510. [CrossRef]

2. Randall, R.B.; Antoni, J.A. Rolling element bearing diagnostics-A tutorial. Mech. Syst. Signal Process. 2011, 25, 485-520. [CrossRef]

3. Rai, A.; Upadhyay, S.H. A review on signal processing techniques utilized in the fault diagnosis of rolling element bearings. Tribol. Int. 2016, 96, 289-306. [CrossRef]

4. Feng, Z.P.; Liang, M.; Chu, F.L. Recent advances in time-frequency analysis methods for machinery fault diagnosis: A review with application examples. Mech. Syst. Signal Process. 2013, 38, 165-205. [CrossRef]

5. Sun, H.L.; He, Z.J.; Zi, Y.Y.; Yuan, J.; Wang, X.D.; Chen, J.L.; He, S.L. Multiwavelet transform and its applications in mechanical fault diagnosis-A review. Mech. Syst. Signal Process. 2014, 43, 1-24. [CrossRef]

6. Lei, Y.G.; Lin, J.; Zuo, M.J.; He, Z.J. Condition monitoring and fault diagnosis of planetary gearboxes: A review. Measurement 2014, 48, 292-305. [CrossRef]

7. Wang, Y.X.; Xiang, J.W.; Markert, R.; Liang, M. Spectral kurtosis for fault detection, diagnosis and prognostics of rotating machines: A review with applications. Mech. Syst. Signal Process. 2016, 66-67, 679-698. [CrossRef]

8. McCormick, A.C.; Nandi, A.K.; Jack, L.B. Application of periodic time-varying autoregressive models to the detection of bearing faults. Proc. Inst. Mech. Eng. C 1998, 212, 417-428. [CrossRef]

9. Wang, W.; Wong, A.K. Autoregressive model-based gear fault diagnosis. Trans. ASME J. Vib. Acoust. 2002, 124, 172-179. [CrossRef]

10. Zhan, Y.M.; Jardine, A.K.S. Adaptive autoregressive modeling of non-stationary vibration signals under distinct gear states. Part 1: Modeling. J. Sound Vib. 2005, 286, 429-450. [CrossRef]

11. Zhan, Y.; Jardine, A.K.S. Adaptive autoregressive modeling of non-stationary vibration signals under distinct gear states. Part 2: Experimental analysis. J. Sound Vib. 2005, 286, 451-476. [CrossRef]

12. Zhan, Y.; Mechefske, C.K. Robust detection of gearbox deterioration using compromised autoregressive modeling and Kolmogorov-Smirnov test statistic-Part I: Compromised autoregressive modeling with the aid of hypothesis tests and simulation analysis. Mech. Syst. Signal Process. 2007, 21, 1953-1982. [CrossRef]

13. Endo, H.; Randall, R.B. Enhancement of autoregressive model based gear tooth fault detection technique by the use of minimum entropy deconvolution filter. Mech. Syst. Signal Process. 2007, 21, 906-919. [CrossRef]

14. Wang, X.; Makis, V. Autoregressive model-based gear shaft fault diagnosis using the Kolmogorov-Smirnov test. J. Sound Vib. 2009, 327, 413-423. [CrossRef]

15. Cong, F.; Chen, J.; Dong, G. Research on the order selection of the autoregressive modeling for rolling element bearing diagnosis. Proc. Inst. Mech. Eng. C 2010, 224, 2289-2297. [CrossRef]

16. Braun, S. The synchronous (time domain) average revisited. Mech. Syst. Signal Process. 2011, 25, 1087-1102. [CrossRef]

17. Klein, R.; Rudyk, E.; Masad, E.; Issacharoff, M. Emphasizing bearing tones for prognostics. Int. J. Cond. Monit. 2011, 1, 73-78. [CrossRef]

18. Battat, M.; Klein, R.; Bortman, J. Application of advanced signal separation methods in a complex case of a bearing fault. Insight 2014, 56, 434-438. [CrossRef]

19. Antoni, J.; Randall, R.B. Unsupervised noise cancellation for vibration signals: Part I-Evaluation of adaptive algorithms. Mech. Syst. Signal Process. 2004, 18, 89-101. [CrossRef]

20. Antoni, J.; Randall, R.B. Unsupervised noise cancellation for vibration signals: Part II-A novel frequency-domain algorithm. Mech. Syst. Signal Process. 2004, 18, 103-117. [CrossRef] 
21. Randall, R.B.; Sawalhi, N.; Coats, M. A comparision of methods for separation of deterministic and random signals. Int. J. Cond. Monit. 2011, 1, 11-19. [CrossRef]

22. Sawalhi, N.; Randall, R.B. Signal Pre-whitening using cepstrum editing (liftering) to enhance fault detection in rolling element bearings. In Proceedings of the 24th International Congress on Condition Monitoring and Diagnostics Engineering Management, Stavanger, Norway, 30 May 2011.

23. Borghesani, P.; Pennacchi, P.; Randall, R.B.; Sawalhi, N.; Ricci, R. Application of cepstrum pre-whitening for the diagnosis of bearing faults under variable speed conditions. Mech. Syst. Signal Process. 2013, 36, 370-384. [CrossRef]

24. Ming, A.B.; Zhang, W.; Qin, Z.Y.; Chu, F.L. Envelope calculation of the multi-component signal and its application to the deterministic component cancellation in bearing fault diagnosis. Mech. Syst. Signal Process. 2015, 50-51, 70-100. [CrossRef]

25. McDonald, G.L.; Zhao, Q.; Zuo, M.J. Maximum correlated kurtosis deconvolution and application on gear tooth chip fault detection. Mech. Syst. Signal Process. 2012, 33, 237-255. [CrossRef]

26. Yang, W.X.; Little, C.; Court, R. S-Transform and its contribution to wind turbine condition monitoring. Renew. Energy 2014, 62, 137-146. [CrossRef]

27. Combet, F.; Gelman, L.; LaPayne, G. Novel detection of local tooth damage in gears by the wavelet bicoherence. Mech. Syst. Signal Process. 2012, 26, 218-228. [CrossRef]

28. Stockwell, R.G.; Mansinha, L.; Lowe, R.P. Localization of the complex spectrum: The S transform. IEEE Trans. Signal Process. 1996, 44, 998-1001. [CrossRef]

29. Fault Data Sets. Available online: http://www.mfpt.org/ (accessed on 10 April 2013).

30. Li, R.Y.; Sopon, P.; He, D. Fault features extraction for bearing prognostics. J. Intell. Manuf. 2012, 23, 313-321. [CrossRef]

31. Zhang, X.H.; Kang, J.S.; Bechhoefer, E.; Zhao, J.M. A new feature extraction method for gear fault diagnosis and prognosis. Eksploat. I Niezawodn. Maint. Reliab. 2014, 16, 295-300.

32. Chaari, F.; Baccar, W.; Abbes, M.S.; Haddar, M. Effect of spalling or tooth breakage on gear mesh stiffness and dynamic response of a one-stage spur gear transmission. Eur. J. Mech. A/Solids 2008, 27, 691-705. [CrossRef]

33. Feng, Z.P.; Zuo, M.J. Vibration signal models for fault diagnosis of planetary gearboxes. J. Sound Vib. 2012, 331, 4919-4939. [CrossRef]

(C) 2017 by the authors. Licensee MDPI, Basel, Switzerland. This article is an open access article distributed under the terms and conditions of the Creative Commons Attribution (CC BY) license (http:/ / creativecommons.org/licenses/by/4.0/). 